\title{
Electronic Structure and Phase Equilibria in Ternary Substitutional Alloys
}

\author{
A. J. S. Traiber \\ P. E. A. Turchi \\ R. M. Waterstrat \\ S. M. Allen
}

RECEIVED

SEP 201996

OSTI

April 26, 1996

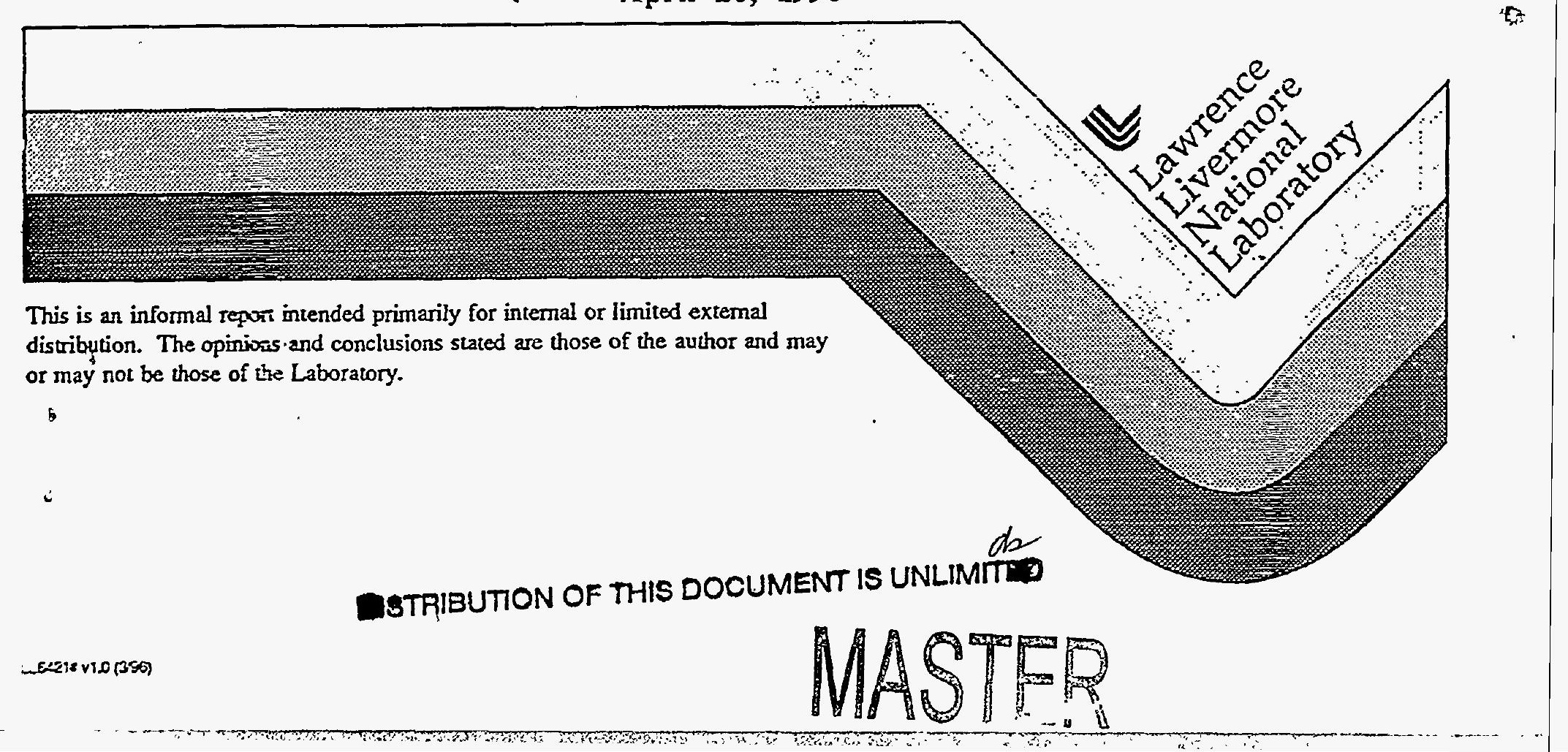




\section{DISCLAIMER}

This report was prepared as an account of work sponsored by an agency of the United States Government. Neither the United States Government nor any agency thereof, nor any of their employees, make any warranty, express or implied, or assumes any legal liability or responsibility for the accuracy, completeness, or usefulness of any information, apparatus, product, or process disclosed, or represents that its use would not infringe privately owned rights. Reference herein to any specific commercial product, process, or service by trade name, trademark, manufacturer, or otherwise does not necessarily constitute or imply its endorsement, recommendation, or favoring by the United States Government or any agency thereof. The views and opinions of authors expressed herein do not necessarily state or reflect those of the United States Government or any agency thereof. 


\section{DISCLAIMER}

Portions of this document may be illegible in electronic image products. Images are produced from the best available original document. 


\title{
Electronic Structure and Phase Equilibria in Ternary Substitutional Alloys
}

\author{
A. J. S. Traiber \\ Department of Materials Science and Engineering, \\ Massachusetts Institute of Technology, Cambridge, MA 02139.
}

P. E. A. Turchi

Chemistry and Materials Science Department, L-268,

Lawrence Livermore National Laboratory, P.O. Box 808, Livermore, C.A 94551.

\author{
R. M. Waterstrat \\ Metallurgy Division, \\ National Institute of Standards and Technology, Gaithersburg MD 20899. \\ S. M. Allen \\ Department of Materials Science and Engineering, \\ Massachusetts Institute of Technology, Cambridge, MA 02139.
}

(April 26, 1996)

\begin{abstract}
A reliable and consistent scheme to study phase equilibria in ternary substitutional alloys based on the tight-binding approximation is presented. With the electronic parameters obtained from linear muffin-tin orbital calculations, we show that the computed density of states and band structures compare well with those obtained from more accurate $a b$ initio calculations. Disordered


alloys are studied within the tight-binding coherent-potential approximation formalism extended to multi-component alloys. The energetics of ordered systems is obtained through effective pair interactions computed with the general perturbation method. Finally, partially ordered alloys are studied with a novel simplification of the molecular coherent-potential approximation combined with the general perturbation method.

The formalism is applied to the study of bcc-based ternary $\mathrm{Zr}-\mathrm{Ru}-\mathrm{Pd}$ alloys which are promising candidates for medical implant device applications. Using the energetics obtained with the aforementioned scheme, we apply the cluster-variation method to study phase equilibria for particular pseudo-binary alloys, and show that the results are consistent with the observed behavior of the electronic specific heat coefficient with composition for the $\mathrm{Zr}_{0.5}(\mathrm{Ru}, \mathrm{Pd})_{0.5}$ system.

64.60.Cn, 71.20.Cf, 81.30.Bx

Typeset using REVTEX 


\section{INTRODUCTION}

Solid solutions with ordered phases ("intermetallic compounds") that exhibit desirable mechanical properties such as ductility and high strength are promising candidates for special applications. Possible uses range from the high-temperature alloys for gas turbines to bearing surfaces and mechanical joints. Because materials designed for optimum performance rarely consist of binary systems, the ability to model higher-order systems is especially needed. In order to develop these materials, the fundamental physical issues must be understood. Theories capable of predicting the type of ordering, the existence of structural transformations, and the phase equilibria are fundamental tools in this endeavor. In the last decade, there has been considerable improvement in the calculation of both energies of formation of disordered and ordered alloys, and multisite effective interactions based on band structure calculations. Such energetic quantities can be used to obtain fairly accurate predictions of phase stability, or ground-states, at $T=0 \mathrm{~K}$. The effective interactions may then be used in combination with statistical models for phase diagram determination.

Any theoretical study of ordering and phase stability in substitutional alloys, and ultimately of their phase diagrams, must begin with reliable and accurate expressions for the energy and entropy as functions of alloy composition and temperature. Over the last few years it has been possible to combine "first-principles" electronic structure calculations for alloy energetics with statistical mechanics-methods for the entropy. These statistical mechanics-methods are based on the so-called generalized Ising model within various approximations. They range from the simpler Bragg-Williams (BW) approximation ${ }^{1}$ to the more sophisticated cluster-variation method (CVM) ${ }^{2}$ and Monte Carlo simulations ${ }^{3}$. In these models it is assumed that the internal energy can be written as a rapidly convergent sum of pair and multisite interactions. Several approaches have been developed to provide the link between electronic structure calculations and statistical models, among them the embeddedcluster method $(\mathrm{ECM})^{4}$, the generalized perturbation method $(\mathrm{GPM})^{5}$, the concentration wave approach ${ }^{6}$, the Connolly-Williams method $(\mathrm{CWM})^{\tau}$, and the direct configurational 
averaging method (DCA) .

To date, applications of these electronic structure and statistical mechanics methods have been mostly confined to binary alloys. Ternary and higher-order alloys, by contrast, remain relatively unexplored. The BW method has been used to study ordering in bcc ternary alloys $^{9-12}$ with special emphasis on the Heusler structure ${ }^{13-16}$. and in fcc lattices ${ }^{17}$. In these examples, "canonical" EPI's were used to study general trends, or were inferred experimentally for more quantitative studies. Kikuchi et al. ${ }^{18}$ studied the fcc $\mathrm{Cu}-\mathrm{Ag}-\mathrm{Au}$ phase diagram using the CVM method in the tetrahedron approximation. Colinet et. al. ${ }^{19}$ reported a similar study on phase equilibria in bcc $\mathrm{Fe}-\mathrm{Co}-\mathrm{Al}$ alloys using the irregular tetrahedron. Examples of Monte Carlo simulations can be found in a review by Inden and $\mathrm{Pisch}^{20}$, and $\mathrm{Traiber}^{21}$, who studied phase equilibria in the bcc $\mathrm{Ni}-\mathrm{Al}-\mathrm{Ti}$ alloys. In all cases, concentration-independent interactions estimated from experimental results were used. More recently. the DCA method has been used to compute interactions to study site substitutions in fcc Ni-Al-X alloys ${ }^{22}$, where $\mathrm{X}=\mathrm{Co}, \mathrm{Cu}, \mathrm{Zn}, \mathrm{Pd}, \mathrm{Si}$, and effective interactions and formation energies for fcc-based Rh-V-Ti, Pd-Rh-V and Ag-Pd-Rh alloys ${ }^{23}$.

This work will focus on one particular alloy theory to study multi-component alloys, namely the GPM implemented within the tight-binding (TB) approximation. In the GPM, a perturbation treatment is applied to a reference medium which is close to any particular configuration of the alloy. Hence, intuitively, the appropriate reference medium to use is the completely disordered state, as the one described by the coherent-potential approximation (CPA, see section III A) ${ }^{5,24,25}$. This TB-CPA-GPM scheme combined with the CVM has been successfully applied to binary alloys. For instance, Sluiter et al. ${ }^{26}$ used this approach to study phase equilibria in Ti-Rh and Ti-Ir alloys with only $d$-orbitals. Sluiter and Turchi ${ }^{27}$ carried out an investigation of phase equilibria in $\mathrm{Ti}-\mathrm{V}$ and $\mathrm{Ti}-\mathrm{Cr}$ alloys. Their study accurately reproduces the energetic properties of Ti-V alloys and provides insight in the possible metastables phases in the Ti-Cr system. Colinet and Pasturel $^{28}$ presented a phase diagram for $\mathrm{Ni}-\mathrm{Ti}$ in good agreement with the experimental one (they used the Cluster Bethe Lattice Method for the liquid phases). Rubin and Finel ${ }^{29}$ reported phase diagram 
calculations for three ternary systems (Ti-Al-Mo, Ti-Al-Nb and Ti-Al-II) using the CPAGPM-CVM approach to write the free energy, but all the parameters (disordered energies and effective interactions) were obtained by a fitting procedure applied to binary phase diagrams. with the assumption that the energetic parameters do not change significantly with the ternary addition. This rough approximation led to a ternary phase diagram in poor agreement with the assessed one. To our knowledge, no study of ternary systems have been carried out using the method to the full extent that is now possible.

We present in this paper a reliable and consistent formalism, based on the TB approximation, to study the electronic structure and phase stability of multi-component transitionmetal alloys. We show how this simple scheme can be used to guide the design of materials with specific electronic properties. First, we characterize the TB parameters computed with the linear muffin-tin orbital method, with direct application to the $\mathrm{Zr}-\mathrm{Ru}-\mathrm{Pd}$ alloy. The CPA and the GPM which are used to study disordered alloy's and ordering phenomena, respectively, are then briefly presented. We then describe an approximation to study partially ordered systems. Results for the ternary $\mathrm{Zr}-\mathrm{Ru}-\mathrm{Pd}$ and its binary subsystems will illustrate the methodology.

The energetic parameters obtained with this methodology will be used in combination with the CVM to study phase equilibria in pseudo-binary alloys. The formalism will be illustrated with the $\mathrm{Z}_{\mathrm{r}_{0.5}}(\mathrm{Ru} . \mathrm{Pd})_{0.5}$ alloy. From these results, we propose an explanation for the observed behavior of the electronic specific heat coefficient $\gamma$.

As we mentioned before, we will illustrate the formalism presented in this paper with the ternary $\mathrm{Zr}-\mathrm{Ru}-\mathrm{Pd}$ alloy. This system is one of a family of alloys under study with possible applications as medical implant devices. For example there are already surgical implants which are made in part of $\mathrm{Co}-\mathrm{Cr}-\mathrm{Mo}$ alloys. The $\mathrm{Zr}-\mathrm{Ru}-\mathrm{Pd}$ alloys have attracted interest because of potentially good biocompatibility. In addition, preliminary experimental work ${ }^{30,31}$ has shown that $\mathrm{Zr}_{0.5}(\mathrm{Ru}, \mathrm{Pd})_{0.5}$ alloys are extremely tough and wear resistant, properties which are highly desirable for implant device applications. The $\mathrm{Zr}-\mathrm{Pd}$ alloy crystallizes at intermediate compositions with a $\beta$-brass (or $B 2$-type) structure but undergoes a martensitic 
transformation at $620^{\circ} \mathrm{C}$; this binary alloy lacks ductility at room temperature. The addition of $\mathrm{Ru}$ seems to stabilize the high-temperature $B 2$ phase at room temperature and significant ductility has been recently reported in these ternary alloys ${ }^{30}$. The equiatomic $\mathrm{Ru}-\mathrm{Zr}$ alloy also forms a B2-type structure which is stable up to its melting point. Ruthenium seems likely to substitute for palladium-atoms in the ternary $B 2$ alloy:

From the experimental work reported on the pseudo-binary $\mathrm{Zr}_{0.5}(\mathrm{Ru}, \mathrm{Pd})_{0.5}$ alloy ${ }^{30}$, a significant extension of the $B 2$ binary phase was found up to about 70 at.\% $\mathrm{Pd}$ before a martensitic transformation takes place, with a sharp minimum of hardness just prior to the onset of the transformation at room temperature. The structure of the martensite was found to be of $B_{f}$ or $B 33$-type, and experimental observations suggest a structural relationship between matrix and martensite which involves two kinds of shuffle-type displacive operations that take the $B 2$ structure into $B 19$ and finally into $B 33^{31}$.

The rest of this paper is organized as follows. In section II, we describe in detail the scaling of the tight-binding parameters obtained from the $a b$ initio calculations. To assess the reliability of these parameters we compare the density of states and the band structures which are obtained with them, to the ones given by the first-principles computations. Section III is concerned with a brief review of the formalism of the electronic structure methods, CPA and GPM, including their extension to study partially ordered alloys. In section IV we present and discuss the results for the binary $\mathrm{Zr}-\mathrm{Ru}, \mathrm{Ru}-\mathrm{Pd}$, and $\mathrm{Zr}-\mathrm{Pd}$ alloys, and for the ternary $\mathrm{Zr}-\mathrm{Ru}-\mathrm{Pd}$ alloys. In section $\mathrm{V}$, the energetic parameters are used in combination with the CVM to study phase equilibria in pseudo-binary alloys. Finally, from these results, we propose in section VI an explanation for the observed variation of the electronic specific heat coefficient with composition for the $\mathrm{Zr}_{0.5}(\mathrm{Ru}, \mathrm{Pd})_{0.5}$ pseudo-binary alloy, before adding some concluding remarks. 


\section{THE TIGHT BINDING PARAMETERS}

\section{A. Background}

Until the seminal paper by Slater and Koster ${ }^{32}$, transfer integrals were computed analytically involving cumbersome integrals that included the atomic orbitals and potentials ${ }^{\mathbf{3 3} 34}$. Slater and Koster derived a method where the TB parameters were to be regarded as adjustable parameters determined by a fit to calculated energy-eigenvalues at various points in the Brillouin zone. These energy values are now computed with more accurate densityfunctional methods like the augmented plane-wave method or APW (see for example ${ }^{35}$ ).

Other approaches, mostly used in conjunction with realistic computer simulations, rely on alternative semi-empirical TB-models. These models include a number of parameters which are obtained by fitting to some experimental values such as cohesive energies, lattice constants and independent elastic constants. These models range from the simple secondmoment approximation to more sophisticated multi-parameter exponential and polynomial functionals ${ }^{36-39}$.

The approach we propose for obtaining the TB parameters uses the TB formulation of the LMTO Hamiltonian, in the atomic sphere approximation (ASA), developed by Anderson et $a l .{ }^{40}$. The $a b$ initio nature of the TB-LMTO approach makes empirical fitting unnecessary for obtaining the Slater-Koster (SK) or fundamental transfer integrals. Also, unlike the fitted SIK parameters, the TB-LMTO SK parameters are defined with reference to the Coulomb potential so that no arbitrary rigid shift of the on-site energies is needed when alloys are considered.

\section{B. Parameters for metals and alloys}

We have mentioned that the hopping integrals can be written in terms of SK parameters which depend on the occupation of sites $n$ and $m$ and the distance joining the two sites. This relation can be written $a s^{32}$ 


$$
\beta_{i(n) j(m)}^{\lambda \mu}=\sum_{h} c_{n m}^{h} w_{i j}^{h}\left(\mathbf{R}_{n, m}\right)
$$

where $w_{i j}^{h}\left(r_{n, m}\right)$ are the SK parameters, the coefficients $c_{n m}^{h}$ depend on the direction cosines of vectors $R_{n, m}$ and $i(n)$ and $j(m)$ show the explicit dependence on the species at sites $n$ and $m$ ( $i, j=A . B$ or $C$ in a ternary alloy). The superscript $h$ runs over the ten possible fundamental SK parameters since $s p d$-electrons are considered, i.e., $h=s s \sigma, p p \sigma, \ldots, d d \sigma, d d \pi, \ldots$, etc.

It is well known that the SK parameters depend on interatomic distance. Our approach is to fit exponential curves to the SK parameters obtained from TB-LMTO calculations for the pure elements at different volumes and lattice structures (bcc and $f c c$ ). It has been shown that atomic environment has little influence on the potential parameters ${ }^{41}$. Figure 1 shows the fit in the case of $\mathrm{Zr}$. The fitting curves are of the form

$$
w_{i}^{h}=\mathcal{A}_{i}^{h} e^{-\mathcal{P}_{i}^{h} d},
$$

where $d$ is the interatomic distance in a.u., $\mathcal{A}_{i}^{h}$ and $\mathcal{P}_{i}^{h}$ are the fitting parameters, and $i$ refers to the species ( $\mathrm{r}$ in this case). Similar results were obtained for $\mathrm{Ru}$ and $\mathrm{Pd}$. In Fig. 1, the interatomic distance was normalized to a reference distance, $d_{o}$, in this case, the equilibrium nearest-neighbor distance in the ordered $B 2 \mathrm{RuZr}$ structure as computed with the LMTO-ASA method: $d_{o}=(\sqrt{3} / 2) a_{B 2}^{\mathrm{RuZr}}=5.3503$ a.u.. Table I gives the parameters computed for the three elements.

The SK parameters for a particular alloy were approximated according to

$$
\beta_{\text {alloy }}^{h}=\left(\sum_{i} c_{i} \sqrt{\beta_{i i}^{h}}\right)^{2},
$$

where $c_{i}$ is the concentration of species $i$ in the alloy. (We have changed the notation of SK parameters to $\beta$ as it is customary.) This equation is arrived at when averaging the hopping integrals for all $i j$ bonds in random alloys, assuming that the hopping between unlike atoms is given by the geometric mean of the appropriate integrals for the pure elements, $\beta_{i j}^{h}=\sqrt{\beta_{i i}^{h} \beta_{j j}^{h}}$ (the so-called Shiba approximation ${ }^{42}$ ). The equilibrium interatomic distance for the alloy case is obtained from the concentration-average of the atomic volumes for the pure species (also known as Zen's law): 


$$
\bar{\Omega}=\sum_{i} c_{i} \Omega_{i} .
$$

This is a reasonable approximation for the present case. Indeed, the equilibrium atomic volume for the (ordered) $\mathrm{ZrRu}$ alloy, taken as an example, computed with the LMTO-ASA method is about $2 \%$ lower than the ideal value given by Eq. 2.4 . In the present case the equilibrium lattice constants for bcc-based $\mathrm{Zr}, \mathrm{Ru}$, and $\mathrm{Pd}$ take the values 6.71267, 5.801941, and 5.870511 a.u., respectively, as obtained from LMTO-ASA. These values will be used to define the lattice constant of alloys based on these three elements at any composition.

\section{On-site energies}

Usually the variation of the on-site energies with atomic volume is ignored in electronic structure calculations. Our LMTO calculations clearly showed a significant variation that should be taken into account. In this work, the on-site energies were extracted from a polynomial fit to first-principles data at different atomic volumes. A common energy shift to all on-site energies associated with the four orbital symmetries, and for each species, was added to obtain local neutrality for the bcc-based $\mathrm{ZrPd}$ and $\mathrm{ZrRu}$ disordered alloys using the CPA approximation (described in the next section). Thus, the on-site energies are given by

$$
\epsilon_{i}^{\lambda}=\epsilon_{i(0)}^{\lambda}+\delta_{i},
$$

where the index 0 denotes the on-site energy associated with $\lambda=s, p, t 2 g, e g$ for the pure element $i$, and $\delta_{i}$ is the energy-shift. In our particular case, the shifts used were $\delta_{\mathrm{Zr}}=0.0$ $\mathrm{Ry}, \delta_{\mathrm{Ru}}=0.3130 \mathrm{Ry}$ and $\delta_{\mathrm{Pd}}=0.3673 \mathrm{Ry}$. When using these values in the computation of the $\mathrm{RuPd}$ random alloy, the resulted charge transfer is about 0.1 electron/atom. Figure 2 shows the fitting for the on-site energies corresponding to Zr. Similar results were obtained for $\mathrm{Ru}$ and $\mathrm{Pd}$. We used a second-order polynomial of the form

$$
\epsilon_{i(0)}^{\lambda}=A_{i}^{\lambda}+B_{i}^{\lambda} a+C_{i}^{\lambda} a^{2}
$$


where $a$ is the lattice constant expressed in atomic units, and calculated from Eq. 2.4, with $\bar{\Omega}=a^{3} / 2$. for bcc-based alloy's. Table II gives the polynomial coefficients for the three elements.

\section{Discussion on the tight-binding approximation}

Before embarking on the study of the chemically disordered alloys it is important to analyze the approximation we have introduced and how the results would differ from the ones obtained from the more accurate first-principles LNTO calculations. To illustrate this point we will compare the band structures and DOS's for an ordered B2 'ZrRu alloy: Our "exact" results correspond to the LMTO calculation of the B2-type structure. We will call it case (a). Our approximation, case (b), uses the tight-binding parameters for the pure elements using the TB-LMTO scheme, and leads to the definition of an average alloy with the SK parameters given by Eq. 2.3 with no off-diagonal disorder.

Figures 3 shows the band structure for both cases. Note that the main features of the band structure are well reproduced by the approximate scheme, especially below the Fermi energy. Figure 4 compares the total DOS for the B2 $\mathrm{ZrRu}$ alloy computed with the LMTO scheme with no off-diagonal disorder to the DOS that corresponds to case (b) in Fig. 3. In the latter case, the detailed features of the DOS, which is computed with the recursion method $^{43,44}$ and 21 levels of continued fraction, are well reproduced. This shows that an accurate and consistent description of the electronic structure (in terms of band structure and DOS) can be achieved within the TB framework. Based on these results we will use this simpler approximation in the rest of this work.

We have described the tight-binding approximation placing special emphasis on the TB parameters. The parameters are extracted form LMTO-ASA calculations and scaled with a novel scheme. We found a good agreement between the TB and the $a b$ initio results of band structures and density of sates. The following sections deal with the energetics of binary and ternary alloys within this TB framework. 


\section{ELECTRONIC STRUCTURE METHODS}

The total energy of an alloy characterized by some ordered structure can be decomposed as follows:

$$
E=\sum c_{i} E_{i}\left(N_{e}^{i}\right)+\Delta E_{d i s}\left(\bar{N}_{e}\right)+\Delta E_{\text {ord }}\left(\overline{N_{e}}\right)
$$

where $E_{i}$ and $N_{e}^{i}$ are the energy and the number of valence electrons per atom of the pure element $i$, respectively. $\Delta E_{d i s}\left(\bar{N}_{e}\right)$ is the energy of the totally disordered state,

$$
\Delta E_{d i s}\left(\bar{N}_{e}\right)=E_{d i s}\left(\bar{N}_{e}\right)-\sum c_{i} E^{i}\left(N_{e}^{i i}\right)
$$

also known as the energy of mixing, and $\Delta E_{\text {ord }}\left(\bar{N}_{e}\right)$ is the oidering energy

$$
\Delta E_{\text {ord }}\left(\bar{N}_{e}\right)=E_{\text {ord }}\left(\bar{N}_{e}\right)-E_{\text {dis }}\left(\bar{N}_{e}\right) .
$$

Notice that $\bar{N}_{e}$ is the average number of electrons, $\bar{N}_{e}=\sum c_{i} N_{e}^{i}$ and that the energy of each element is computed with its own Fermi energy $E_{F}^{i}$ (associated with $N_{e}^{i}$ ). Finally the formation energy is defined as:

$$
\Delta E_{\text {form }}=\Delta E_{\text {dis }}+\Delta E_{\text {ord }} .
$$

The following sub-sections deal with the computation of the disordered and ordering energies introduced in Eq. 3.1.

\section{A. Disordered Alloys and the Coherent-Potential Approximation}

The coherent-potential approximation (CPA $)^{45}$ is a mean-field theory that describes the average electronic structure of the completely disordered state of an alloy. It is considered as the best single-site approximation for treating random alloys. In the absence of shortrange order, the constituents of the alloy are placed randomly on the periodic underlying lattice, thus Bloch's theorem does not apply. The CPA restores the translational invariance by defining an average medium, represented by a self-energy (or coherent potential) $\sigma$. For 
each orbital $\lambda$, the potential $\sigma^{\lambda}$ is located at all sites except the central one, which is occupied by an on-site energy associated with species $i$ and orbital $\lambda$. Thus, this fixed atom is assumed to be embedded in the uniform medium of the disordered state, and as such the CPA is a single-site approximation. This central atom causes scattering of electrons by the potential difference $\Delta \epsilon_{i(0)}^{\lambda}(z)=\epsilon_{i(0)}^{\prime}-\sigma^{\lambda}(z)$ between the potential at site 0 occupied with atom $i$ and that of the uniform medium. Here, $z$ is the energy plus an infinitesimal imaginary part. We aiso assume that the alloy is homogeneous, i.e., that all the sites are equivalent and $\sigma$ does not depend on the site $n$, but we allow for multiple orbitals $\lambda$. The scattering can be described by the $t$-matrix element

$$
t_{i}^{\lambda}=\frac{\Delta \epsilon_{i(0)}^{\lambda}(z)}{1-\Delta \epsilon_{i(0)}^{\lambda}(z) G_{00}^{\lambda \lambda}(z)}
$$

where $G_{00}^{\lambda \lambda}$ is the diagonal matrix element of the Green's function for the random medium, $\bar{G}$. The coherent potential $\sigma^{\lambda}$ can be obtained self-consistently if we require that the scattering 'on average' vanishes. For a ternary alloy we have:

$$
\left\langle t^{\lambda}\right\rangle=c_{A} t_{A}^{\lambda}+c_{B} t_{B}^{\lambda}+c_{C} t_{C}^{\lambda}=0
$$

The notation $\langle\cdots\rangle$ is taken to be the so-called configurational average which is the average over all possible atomic configurations on the lattice sites at the average concentration of the alloy. Since $t_{\mathrm{i}}^{\lambda}$ is a function of $z, \epsilon^{\lambda}$ and $\bar{G}$, the self-energy $\sigma^{\lambda}$ must be found iteratively. The first guess for the coherent potential is given by the Virtual Crystal Approximation (or VCA) value, $\sigma_{0}^{\lambda}=\sum c_{i} \epsilon_{i}^{\lambda}$. To formally describe the coherent potential and $\bar{G}(z)$, we start with the Hamiltonian of the alloy written as follows,

$$
\begin{aligned}
H & =W+V \\
W & =\sum_{m n, \mu \lambda}|n, \lambda\rangle \beta_{n m}^{\lambda \mu}\langle m, \mu| \\
V & =\sum_{n, \lambda}|n, \lambda\rangle \epsilon_{n}^{\lambda}\langle n, \lambda| .
\end{aligned}
$$

The hopping integrals $\beta_{n m}^{\lambda \mu}$ are assumed to be independent of the nature of the atoms located at sites $n$ and $m$, and $W$ is then the translationally invariant part of the alloy Hamiltonian. The $\epsilon_{\pi}^{\lambda}: s$ are distributed randomly on the lattice and are defined as 


$$
\epsilon_{n}^{\lambda}=\sum_{i} p_{n}^{i} \epsilon_{i}^{\lambda}
$$

where the occupation number $p_{n}^{i}=1$ if atom of type $\mathrm{i}$ occupies site $n$. otherwise $p_{n}^{i}=0$.

The Green's function for a particular configuration is given by $G(z)=(z-H)^{-1}$, and the self-energy $\Sigma(z)$ is then defined for the average Green's function $\bar{G}$ as

$$
\bar{G}(z)=\langle G(z)\rangle=(z-V-\Sigma(z))^{-1} .
$$

Within the CPA, the resulting self-energy is site-diagonal, i.e.,

$$
\Sigma(z)=\sum_{n, \lambda}|n, \lambda\rangle \sigma^{\lambda}\langle n, \lambda|
$$

Equation 3.6 actually represents nine equations that must be solved simultaneously. The average Green's function is given by an integration in reciprocal space over the first Brillioun zone (BZ) performed with a technique of special k-points ${ }^{46}$,

$$
\bar{G}(z)=\frac{1}{\Omega_{\mathrm{BZ}}} \int_{\mathrm{BZ}}(z-\sigma-W(\mathrm{k}))^{-1} \mathrm{~d}^{3} \mathrm{k},
$$

where $W(k)$ is the Fourier transform of $W$ and $\Omega_{\mathrm{BZ}}$ is the volume of the first BZ. Partial densities of states are obtained from the Green's function according to

$$
n_{i}^{\lambda}(E)=-\frac{2}{\pi} \lim _{\eta \rightarrow 0^{+}} \operatorname{Im}\langle 0 \lambda|\bar{G}(E+i \eta)| 0 \lambda\rangle,
$$

whereas band energies are given by

$$
E_{b}^{C P A}=\int_{-\infty}^{E_{F}} E n(E) \mathrm{d} E
$$

Here $n(E)$ is the total density of states, $n(E)=\sum_{i, \lambda} c_{i} n_{i}^{\lambda}(E)$ and $E_{F}$ is the Fermi energy associated with the CPA medium. The factor 2 in Eq. 3.14 accounts for the spin degeneracy.

\section{B. Ordered Alloys}

Ordering processes in alloys are conveniently described by the use of an Ising model. This model was first introduced to study magnetic systems in which each atom of the lattice 
is supposed to have a magnetic moment (Ising spin) $\sigma_{n}$ which can take on one of two possible ralues. Then the Hamiltonian of the system takes the form:

$$
H=-\sum_{n m} J_{n m} \sigma_{n} \sigma_{m}
$$

where $J_{n m}$ is an exchange integral between spins on sites $n$ and $m$ and the spin variable $\sigma_{n}$ is $1(-1)$ if the spin at site $n$ points 'upward' ('downward'). The summation is over interacting neighbor pairs. The same concept to approximate random or partially ordered multicomponent substitutional alloy's can be used by writing the configurational energy (with only pair interactions) as

$$
E=\frac{N}{2} \sum_{i, j=1}^{M} \sum_{n, m=1}^{N} V_{n m}^{i, j} p_{n}^{i} p_{m}^{j}
$$

where $V_{n, m}^{i, j}$ is an effective real-space pair potential between species $i$ and $j$ at sites $n$ and $m$, respectively. There are $N$ sites and $M$ chemical species. The factor $1 / 2$ is required to avoid double counting. One major difference between the alloy and the Ising systems is that the magnetic interactions $J_{n m}$ in the Ising model are postulated to be independent of both temperature and concentration (of spins in either direction). On physical grounds, there is no compelling reason to assume that interatomic interactions in alloys possess these properties $^{47}$, although the temperature dependence of the interactions seems to be most important for magnetic systems ${ }^{20}$. An exhaustive analysis of the approximations involved in the use of this "generalized" Ising model when mapping the real free energy of an alloy is discussed in Ref. 48.

The generalized perturbation method (GPM) $)^{5,25}$ attempts a direct determination of concentration-dependent multi-site interactions in real-space. The method is a perturbation treatment applied to a reference medium which is close to any particular configuration of the alloy, such as the complete disordered state described by the CPA. Any chemical configuration is completely specified by the set of occupation numbers $\left\{p_{n}^{i}\right\}$. For a particular configuration $\left\{p_{n}^{i}\right\}$ the GPM allows the band energy $E\left(\left\{p_{n}^{i}\right\}\right)$ to be expressed (see $e^{25,49}$ for a derivation of the equations) as: 


$$
E\left(\left\{p_{n}^{i}\right\}\right)=E_{\text {dis }}(c)+\Delta E_{\text {ord }}\left(\left\{p_{n}^{i}\right\}\right)
$$

where the energy of the disordered state $E_{d i s}$ is concentration-dependent but independent of the $\left\{p_{n}^{i}\right\}$ (thus, configuration-independent) as calculated with the CPA method, and $\Delta E_{\text {ord }}$ is the ordering energy which can be expanded as follows:

$$
\begin{aligned}
\Delta E_{\text {ord }}= & \frac{1}{2 N} \sum_{\substack{i, j \\
n, m, n \neq m}} V_{n m}^{i j,(2)} \delta c_{n}^{i} \delta c_{m}^{j} \\
& +\frac{1}{3 N} \sum_{\substack{i, k \\
n \neq l, k, j \\
n \neq m, m \neq l, n \neq l}} V_{n m l}^{i j k,(3)} \delta c_{n}^{i} \delta c_{m}^{j} \delta c_{l}^{k}+\cdots
\end{aligned}
$$

where $\delta c_{n}^{i}$ is the concentration deviation from the average composition at site $\mathrm{n}, \delta c_{n}^{i}=$ $p_{n}^{i}-c^{i}$ and $V_{n m}^{i j . . .(l)}$ are the concentration-dependent $l$-site effective cluster interactions between species $i, j, \ldots$ at sites $n, m, \ldots$ Usually, higher-order terms (greater than two) in Eq. 3.19 are negligible. In the following we will only consider the second-order terms which comprise the effective pair interactions (EPI's), redefined as $V_{s}^{i j}$ where $s$ is a shell index. In terms of Green's function matrix elements, these interactions are given by

$$
V_{s}^{i j}=-\frac{1}{\pi} \operatorname{Im} \int^{E_{F}} \mathrm{~d} E \sum_{\lambda \mu} G_{(s)}^{\lambda \mu} G_{(s)}^{\mu \lambda} \Delta t_{i j}^{\lambda} \Delta t_{i j}^{\mu},
$$

where $E_{F}$ is the Fermi energy of the CPA medium, $G_{(s)}^{\lambda \mu}$ is the off-diagonal in site matrix element of the CPA Green's function between sth-neighbors and $\Delta t_{i j}^{\lambda}=t_{i}^{\lambda}-t_{j}^{\lambda}$. Notice that if $V_{s}^{i j}>0$ then clustering of unlike $s$ th-neighbor pairs is favored. Finally, the ordering energy for the ternary system can be expressed as

$$
\Delta E_{\text {ord }}=\frac{1}{2} \sum_{i \neq j, s}\left(c_{i} c_{j} z_{s}-\delta q_{s}^{i j}\right) V_{s}^{i j}=\sum_{i \neq j, s} q_{s}^{i j} V_{s}^{i j},
$$

where $z_{s}$ is the coordination number, $\delta q_{s}^{i j}$ is the number of $i j$ pairs, per atom, associated with the $s$ th shell, and the $q_{s}^{i j}$ are the GPM expansion coefficients.

\section{Partially Random Alloys}

As we mentioned in the Introduction, the stable phase along the tie-line describing the $\mathrm{Zr}_{0.5}(\mathrm{Ru} . \mathrm{Pd})_{0.5}$ pseudo-binary system has a $B 2$ structure beyond the martensitic region. We 
have studied the characteristics of partially random alloys where, for example, $\mathrm{Zr}$ occupies one of the two simple cubic sublattices which constitute the bcc lattice. and Pd and $\mathrm{Ru}$ randomly occupy the other sublattice. Although the present theory is applicable to more complicated compounds, we restrict the following arguments to those particular bcc-based intermetallic compounds, represented by $\left(A_{1-c} B_{c}\right)_{0.5} C_{0.5}$. They consist of two interpenetrating primitive sublattices, $\alpha$ where $A$ and $B$ atoms are distributed randomly, and $\beta$ which is occupied by only $C$ atoms. The values of the on-site energies are given by (we drop the orbital superscript for simplicity),

$$
\epsilon_{n}= \begin{cases}\epsilon_{A} \text { or } \epsilon_{\mathrm{B}} & \text { if } n \in a \\ \epsilon_{C} & \text { if } n \in \beta\end{cases}
$$

A rigorous treatment of this system would require, for example, the use of the two-site cluster CPA (CCPA) (see, for instance, Ref. 50) where the disordered material is considered to be a collection of clusters chosen so that the entire lattice can be generated by the translation of the points in a cluster through a set of translation vectors. Each cluster or cell contains several atoms (two in our case), and we apply the CPA equation to the cell rather than to a single site. The cluster Green's function now becomes a matrix and the scalar CPA self-consistent condition given by Eq. 3.6, is generalized to a matrix self-consistent condition. The CPA self-energy $\sigma$ is replaced by a (18 x 18$)$ cluster diagonal matrix, $\Sigma$.

Wie can rewrite the Hamiltonian matrix of the disordered material in terms of cluster quantities. Let $\underline{\epsilon}_{C}$ denote the cluster-diagonal part of $H$, where $C=\alpha$ or $\beta$, and $\underline{W}_{C C^{\prime}}$ the cluster off-diagonal part. We have then (dropping orbital superscripts)

$$
\left(\underline{\epsilon}_{C}\right)_{i j}=\left\{\begin{array}{l}
\epsilon_{i} \delta_{i j} \\
\beta_{i j}\left(1-\delta_{i j}\right) \quad i, j \in C,
\end{array}\right.
$$

and

$$
\left(\underline{\underline{X}}_{C C^{\prime}}\right)_{i j}=\beta_{i j} \quad i \in C, j \in C^{\prime}, C \neq C^{\prime}
$$

To avoid the tedious calculations involved in the CCPA computations we applied the following approximation. The self-energy of the effective medium is required to be not only 
cluster-diagonal but also site-diagonal, that is $(\Sigma)_{i j}=\sigma \delta_{i j}$. Furthermore, the coherent potential $\sigma$ is placed only on the random sublattice. This approach is similar to the selfconsistent boundary-site approximation of the cluster CPA $\mathrm{A}^{51}$. We impose the additional restriction of fixing the on-site energies in the fully ordered sublattice, and apply the CPA condition on the random sublattice, restoring the scalar nature of the single-site CPA.

For a full spd-electronic system, the $\Sigma$-matrix takes the form

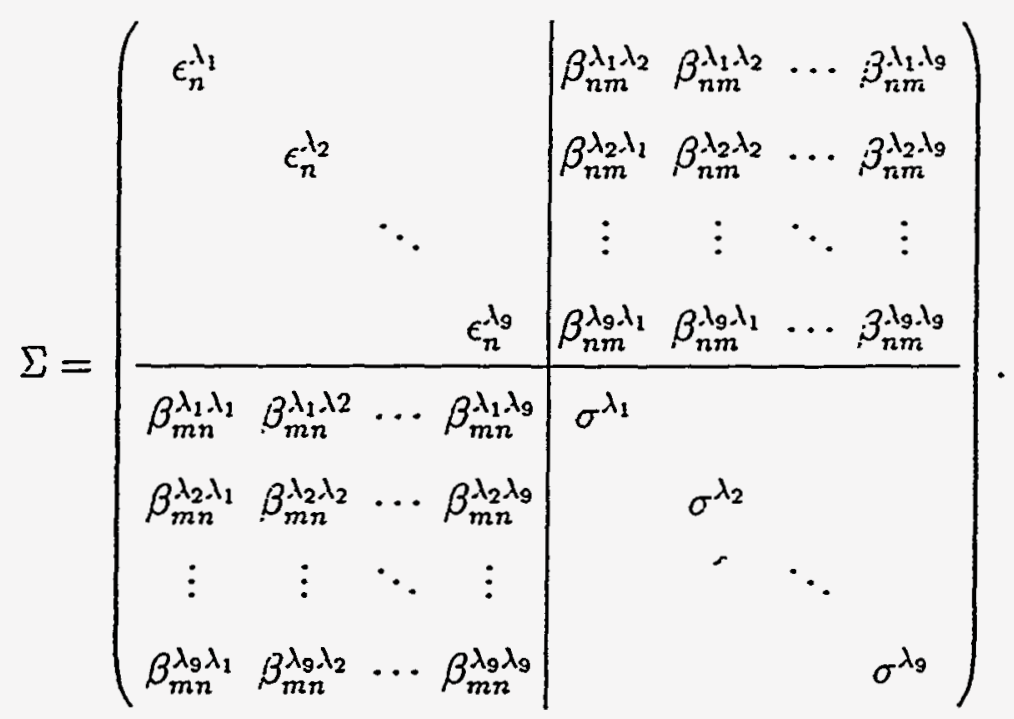

To test this approximation, which we call "partial CPA" (PCPA), we have compared results with the rigorous two-site cluster CPA treatment of the alloy. In Fig. 5, we can see that a good agreement exists between the total DOS's for the $\mathrm{Zr}_{0.5}\left(\mathrm{Ru}_{0.5} \mathrm{Pd}_{0.5}\right)_{0.5}$ alloy computed with both methods. Let us define $\tilde{\epsilon}_{\mathrm{Z}_{r}}^{\lambda}$ as being the site-diagonal element of the effective Hamiltonian resulting from a self-consistent calculation performed within the CCPA, and projected on a site of the fully ordered sublattice occupied by $\mathrm{Zr}$, and of orbital-symmetry $t 2 g$. Figure 6 shows both the potential $\sigma^{t_{2 g}}$ and this on-site term $\hat{\epsilon}_{\mathrm{Zr}}^{t_{2 g}}$ as functions of energy, as computed with the CCPA. Notice the different scaling factors applied to the vertical axis. We obtained similar behavior for the on-site energies associated with $s, p$ and $e g$ symmetries. This figure shows that the fluctuation of the on-site energy for the ordered sublattice is vanishing when compared with respect to the atomic $(\mathrm{Zr})$ on-site energy value, which in the present case is equal to 0.29765 Ry. We can then consider a fixed on-site energy on this ordered sublattice, and apply the partial CPA to the fully random sublattice only. 
The GPM, discussed in the sub-section III.B, can also be applied to partially random alloys. When this is done, the computed interactions are associated with the random simple cubic sublattice. The energy for a particular ternary configuration may then be obtained with two different expansions (see Eq. 3.21):

$$
E_{\text {ord }}=E_{C P A}+\sum_{i \neq j, s} q_{s}^{i j} \bigvee_{s}^{i j}=\tilde{E}_{P C P A}+\sum_{i, j \in a, s} \tilde{q_{s}} \tilde{V_{s}^{i j}}
$$

where the $\tilde{q}_{i}$ and $\tilde{V}_{i}$ are respectively the expansion coefficients and EPI's associated with the specinic sublattice $(\alpha)$ where ordering takes place (here the simple cubic sublattice), and $\tilde{E}_{C P A}$ is the energy of the partially random alloy. We have then two different approaches to study stability and order in multi-component alloys. The first one has the fully random alloy as the starting point, while the second one starts by considering a partially ordered system. These two approaches can be used advantageously to study preferential substitution in multi-component alloys, since the energetics computed in both approaches, depending on the assumption made on preferential substitution, should give compatible results.

\section{RESULTS}

\section{A. Binary Alloys}

Wie studied the electronic structure of bcc-based $\mathrm{Ru}-\mathrm{Zr}, \mathrm{Pd}-\mathrm{Zr}$ and $\mathrm{Ru}-\mathrm{Pd}$ alloys at different compositions using the TB-CPA-GPM formalism. For example, Fig. 7 shows the CPA-DOS's for the three alloys at equiconcentration. They all exhibit a pseudo-gap around the half-filled band with two peaks of strong $d$-character. This is typical for DOS of bcc metals and alloys where the lower half region is related to bonding states and the upper half region, to antibonding states ${ }^{52}$. According to Friedel's theory of cohesive energy for transition metals (TM's), the essential contribution to cohesion in TM-compounds is the broadening of the TM $d$-band; and the occupation of the bonding or antibonding states increases or reduces the cohesion (or stability). In the case of $\mathrm{Ru}-\mathrm{Pd}$ the Fermi energy falls far from the pseudo-gap, lying on a peak of antibonding nature. This may be associated 
to the instability of the bcc Ru-Pd solution (see, e.g., Ref. 53 for similar analysis of TM DOS's). Furthermore Table III shows that the first four nearest neighbor EPI's between Ru and $\mathrm{Pd}$ are negative and, as shown in Fig. Sa, the mixing energy is positive suggesting a clear tendency torrards phase separation. On the other hand we confirm a strong $B 2$ ordering tendency in $\mathrm{RuZr}$ and a weaker $B 2$ ordering tendency in $\mathrm{PdZ}$. The $B 2$ ordering is favored when $F_{1}>0$ and $V_{2} / V_{1}<2 / 3^{54}$. As expected, the mixing energies for both systems are negative as shown in Fig. 8 b.

\section{B. Ternary Alloys}

The tendency towards mixing of the ternary alloy was evaluated with $\Delta E_{\text {mix }}$, defined as

$$
\Delta E_{\operatorname{mix}}\left(\left\{c_{i}\right\}, a\right)=E_{\text {dis }}\left(\left\{c_{i}\right\}, a\right)-\sum_{i} c_{i} E_{i}\left(a_{i}\right)
$$

where $a$ and $a_{i}$ represent, respectively, the lattice parameter of the random alloy and of the pure element $i$. This formula was used to map the energy of mixing on the Gibbs' triangle for the bcc-based $\mathrm{Zr}-\mathrm{Ru}-\mathrm{Pd}$ alloys. We found that the energy of mixing is negative except in a narrow range of concentration close to the $\mathrm{Ru}-\mathrm{Pd}$ side, as expected.

We were interested in the ternary alloy $\mathrm{Zr}_{0.5}\left(\mathrm{Ru}_{1-c} \mathrm{Pd}_{c}\right)_{0.5}$ for which the bulk of experimental data are available. The first and second nearest-neighbor EPI's, $V_{1}$ and $V_{2}$, as obtained from the GPM applied to the fully random alloys, are displayed in Figs. 9, as functions of concentration $c$. Notice that $V_{1}$ is positive and similar in magnitude for $\mathrm{Ru}-\mathrm{Zr}$ and $\mathrm{Pd}-\mathrm{Zr}$ while the interaction between $\mathrm{Pd}$ and $\mathrm{Ru}$ remains negative and about one order of magnitude smaller. A ground-state analysis performed with a cluster method ${ }^{55}$ and which included nearest and next-nearest neighbor interactions predicts a tendency towards phase separation in two $B 2$ phases. This is expected from the magnitude and the negative sign of the EPI's involved, particularly the next nearest-neighbor interaction, $V_{2}^{R u P d}$, between $R u$ and $\mathrm{Pd}$.

Figure 10 shows the variation of this interaction with the filling of the band (or the average number of valence electrons for the alloy). This figure tell us an important result. 
Simple band-filling arguments show that replacing one element like $R u$ by a metal with a lower number of valence electrons could turn this interaction positive without altering the other ones significantly: In this way we may be able to stabilize an ordered structure like the $L 2_{1}$ of Heusler's type. That is possible for an $A_{0.5} B C$ alloy since the ordering energy difference between a Heusler phase, $L 2_{1}\left(A_{2} B C\right)$, and a mixture of two $\beta$ phases, $B 2(A B) / B 2(A C)$, is controlled mainly by $V_{2}^{B C}$. Our preliminary calculations agree with this analysis if $\mathrm{Ru}$ is replaced, for instance, by $\mathrm{Mo}$.

These results motivated the study of partial order in the $\mathrm{Zr}_{0.5}(\mathrm{Ru}, \mathrm{Pd})_{0.5}$ system with the formalism described in sub-section III.C. We applied the PCPA-GPM to a simple cubic sublattice occupied by $\mathrm{Pd}$ and $\mathrm{Ru}$ only. The interactions that we obtained are in agreement with those obtained using the full CPA, i.e., they are all negative so that the elements tend to segregate on this sublattice, giving rise to a phase separation in two $B 2$ phases, as concluded before. Furthermore, the disordered energy for this system is more negative than the disordered energy of the fully random alloy at all concentrations, as shown in Fig. 11. This indicates that the partially random configuration should, in principle, be more stable than the totally random one, which is consistent with a preferential occupation of $\mathrm{Zr}$ on one sublattice, as observed in the $B 2$ structures of $\mathrm{ZrRu}$ and $\mathrm{ZrPd}$.

The following section shows how the energetic parameters obtained within the TB-CPAGPM scheme are used to study phase equilibria in alloys.

\section{PHASE EQUILIBRIA IN PSEUDO-BINARY ALLOYS}

Up to this point, we were only concerned with the energetic properties at zerotemperature. Equilibrium states at finite temperature can be obtained by minimizing the Helmholtz free energy, $F\left(N_{i}, V, T\right)=U-T S$ at fixed number of particles $N_{i}$, since the energetic parameters which enter this functional are, in our case, concentration-dependent. Here, $U$ is the internal energy, $T$ the temperature, $V$ the volume and $S$ the entropy of the system. In this study, the energy of ordering and the configurational entropy are com- 
puted at finite temperature with the CVM, the derivation of which can be found in several publications (see for instance Ref. 56).

The phase equilibria of the ternary $\mathrm{Zr}-\mathrm{Ru}-\mathrm{Pd}$ alloy were limited to the study of the equilibrium properties of the $\mathrm{Zr}_{0.5}(\mathrm{Ru}, \mathrm{Pd})_{0.5}$ system. From the ground-state analysis we expect this ternary system to phase separate in two $B 2$ phases. Thus, we populated one sublattice fully with $\mathrm{Zr}$ and studied the stability of $\mathrm{Ru}-\mathrm{Pd}$ in the second sublattice. We then applied the CVM to this sublattice taking the simple cube as the maximal cluster. Twentyone correlation functions are necessary to describe the equilibrium configuration for the phase separating system in this approximation. Figure 12 shows the results we obtained for this pseudo-binary system using the EPI's $\tilde{V}_{j}^{\text {RuPd }}$ calculated with the GPM applied to the PCPA medium, as described in subsection III.C. The phase diagram displays a miscibility gap at low temperatures, that is a $B 2 / B 2$ phase separation, which is consistent with interactions being all negative. Thus, according to this theoretical investigation, we show that the $\mathrm{Zr}$ species occupies preferentially one of the simple cubic sublattice (see Fig. 11) whereas Ru and $\mathrm{Pd}$ exhibit, on the other sublattice, a strong tendency toward phase separation. On the experimental side, this ternary alloy, along the tie line $\mathrm{ZrRu}$ and $\mathrm{ZrPd}$ has been characterized by $\mathrm{x}$-ray diffraction ${ }^{30,31}$ from $16 \mathrm{~K}$ to room temperature, and a monotonic variation of the lattice parameter with alloy composition has been observed from 0 up to about 70 at.\% Pd where the martensitic transformation takes place. This experimental finding seems to indicate that a solid solution, i.e., a chemically random alloy configuration, exists between $\mathrm{ZrRu}$ and $\mathrm{ZrPd}$, with no existence of a two-phase field as predicted by theory. This apparent contradiction can be resolved if one assumes, starting from the theory side, that because of the strong clustering tendency (see Fig. 12), any alloy derived from the melt will result, in the absence of a kinetic driving force, in a "quench-in" state which can only exhibit shortrange order (SRO), and therefore a single-phase field. The SRO in this alloy case would be difficult to detect since the diffuse scattering associated with the tendency toward phase separation on one of the simple cubic sublattice is located at the Bragg positions of the bcc lattice. In the following section we will see how this tendency toward clustering could 
explain the observed behavior of the electronic specific heat in these alloys.

\section{CALCULATION OF THE ELECTRONIC SPECIFIC HEAT}

If there is one theme we want to emphasize in this paper; it is the close relationship between electronic structure and ordering phenomena in alloøs. One goal is to predict the occurrence and the type of ordering from the knowledge of the electronic structure. At the same time, we want to study how ordering can influence certain electronic properties. One possible macroscopic observable to consider is the linear coefficient of the low-temperature specific heat, $\gamma$, which is related to the properties of the electrons at the Fermi energy. Usually the specific heat of a metal or an alloy obeys the law:

$$
C(T)=\gamma T+\beta T^{3}+\cdots
$$

where the first term is the electronic contribution, and the second term is the lattice (phonon) contribution. The coefficient $\gamma$ turns out to be proportional to the DOS at the Fermi energy $^{59}, n\left(E_{F}\right)$ :

$$
\gamma=\frac{\pi^{2}}{3} k_{b}^{2} n\left(E_{F}\right)
$$

where $k_{b}$ is the Boltzmann's constant. Actually, the electron-phonon coupling $\left(\lambda_{e-p}\right)$ and the spin fluctuations $\left(\lambda_{s f}\right)$ can increase the bare DOS, and $\gamma$ is more precisely given by ${ }^{5 \tau}$

$$
\gamma=\frac{\pi^{2}}{3} k_{b}^{2}\left(1+\lambda_{e-p}+\lambda_{s f}+\cdots\right) n\left(E_{F}\right)
$$

Figure 13 shows the variation of the electronic specific heat coefficient $\gamma$ with composition for the pseudo-binary $\mathrm{Zr}_{0.5}\left(\mathrm{Ru}_{1-c} \mathrm{Pd}_{c}\right)_{0.5}$ as obtained from low-temperature calorimetry ${ }^{58}$. It shows an approximately linear increase of $\gamma$ with composition until it drops around $c=0.68$ where the martensitic transformation takes place. This behavior has been observed in similar B2-type pseudo-binaries. For instance, $\gamma$ for $\mathrm{Ti}_{0.5}(\mathrm{Ni}, \mathrm{Fe})_{0.5}$ presents a rounded peak around the concentration where the martensitic transformation takes place, whereas 
in $\mathrm{Ti}_{0.5}(\mathrm{Ni}, \mathrm{Os})_{0.5}$ and $\mathrm{Ti}_{0.5}(\mathrm{Ni} . \mathrm{Ru})_{0.5}$ the rounded peak is larger and can be thought of as forming a "plateau" like in the $\mathrm{Zr}_{0.5}(\mathrm{Ru}, \mathrm{Pd})_{0.5} \mathrm{case}^{5 \tau}$.

Assuming that the electron-phonon coupling and the spin fluctuations are constant upon alloying, the variation of $\gamma$ with composition is compatible with the results of our electronic structure model. First, let us assume that the alloy exhibits a tendency toward phase separation, as discussed in section V. With such assumption, $\gamma$ for the alloy is well approximated by the concentration-weighted average of $\gamma(\mathrm{RuZr})$ and $\gamma(\mathrm{PdZr})$. Figure 14a shows the $n\left(E_{F}\right)$ that corresponds to a phase separating system for which the total DOS was computed as the concentration-weighted average of the DOS's of the two B2-type binary alloys RuZr and PdZr. Second, using the PCPA, we also calculated the DOS for the partially ordered alloy as a function of composition, and the results are shown in Fig. 14b. In that case, the DOS flattens out in the region of highly concentrated Pd-alloys, and this behavior is attributed to a virtual gap at the Fermi level in the MTRX plane of the simple cubic Brillouin zone. Besides this minor difference with the previous case, the behaviors of the two alloy configurations displayed in Fig. 14 are rather similar and therefore difficult to distinguish experimentally. However the approximately linear increase of $n\left(E_{F}\right)$ with composition is, to some extent, in better agreement with experiment in the case of the pseudo-binary alloy exhibiting a tendency toward phase separation. After conversion to the appropriate units, the rate of increase (taking only the end points) of $\gamma$ agrees to within $15 \%$ of the experimentally determined one.

\section{CONCLUSIONS}

We have described and analyzed an electronic structure method based on the TB approximation to study the energetics of multicomponent alloy's. First, a novel scaling scheme for the TB parameters was introduced. The CPA and GPM were then presented with their natural extension for treating ternary systems. The method allows the study of partially ordered systems using a reliable approximation of the cluster CPA. The methodology was 
then applied to the study of bcc-based $\mathrm{Zr}-\mathrm{Ru}-\mathrm{Pd}$ alloys. The effective interactions for the binary systems give rise to ground-states at equiatomic composition in agreement with the available experimental data. The analysis of the second nearest neighbor EPI, $V_{2}^{\text {RuPd }}$, shows that our predictive capabilities can be used to guide the design of new alloys with specific ordered configurations.

Lising the energetic parameters obtained with the TB-CPA-GPM model, we have applied the Cl'M to compute the bcc-based phase diagrams of the binary $\mathrm{Ru}-\mathrm{Zr}, \mathrm{Pd}-\mathrm{Zr}$, and $\mathrm{Ru}-\mathrm{Pd}$ alloys. and of the pseudo-binary $\mathrm{Zr}_{0.5}(\mathrm{Ru}, \mathrm{Pd})_{0.5}$ alloys. Qualitatively, the equilibrium phases that were obtained for the binary systems agree with the experimental phase diagrams. Based on the $B 2 / B 2$ two-phase field predicted for the ternary alloy along this particular tie-line, we proposed an explanation for the observed variation of the electronic specific heat coefficient, $\gamma$, with composition in $\mathrm{Zr}_{0.5}(\mathrm{Ru}, \mathrm{Pd})_{0.5}$ alloys.

We believe that this TB-CPA approach, which includes the treatment of partially ordered alloys, can be successfully applied to study the electronic origin of the martensitic transformation in $\mathrm{Zr}_{0.5}\left(\mathrm{Ru}_{1-c} \mathrm{Pd}_{c}\right)$ alloys and similar systems, and details of this study will be reported in a forthcoming paper.

\section{ACKNOWLEDGMENTS}

This work was performed under the auspices of the U.S. Department of Energy by the Lawrence Livermore National Laboratory under contract No. W-7405-ENG-48. The authors are grateful to Dr. A. Gonis at LLNL for fruitful discussions. 


\section{REFERENCES}

${ }^{1}$ W. L .Bragg and E. J. Williams. Proc. Roy. Soc. A 145. 699 (1934): 151. 540 (1935); $152,231(1935)$.

${ }^{2}$ R. Kikuchi, Phy's. Rev. 79, 718 (1950): 81, 98 (1951).

${ }^{3} \mathrm{~K}$. Binder, in Monte Carlo Methods in Statistical Mechanics, Topics in Current Physics Vol. 7, edited by K. Binder (Springer, Berlin, 1986), p. 1.

${ }^{4}$ A. Gonis, X.-G. Zhang, A. J. Freeman, P. Turchi, G. M. Stocks, and D. M. Nicholson, Phys. Rev. B 36, 4630 (1987), and refs. therein.

${ }^{5}$ F. Ducastelle and F. Gautier, J. of Phys. F: Mat. Phys. 6. 2036 (1976).

${ }^{6}$ B. L. Györffy and G. M. Stocks, Phys. Rev. Lett. 50, 374 (19S3).

${ }^{7}$ J. W. D. Connolly and A. R. Williams, Phys. Rev. B 27, 5169 (1983).

${ }^{8}$ H. Dreyssé, A. Berera, L. T. Wille, and D. de Fontaine, Phys. Rev. B 39, 2442 (1989).

${ }^{9}$ J. L. Meijering, Philips Res. Rep. 5, 333 (1950); 6, 183 (1951). .

${ }^{10}$ T. Nishizawa, S. M. Hao, M. Hasebe, and K. Ishida, Acta Metall. 31, 1403 (1983).

${ }^{11}$ Masuhiro Fukaya, Toru Miyazaki, Pi Zhi Zhao, and Takao Kozakai, J. Mater. Sci. 25, 522 (1990).

${ }^{12}$ Masuhiro Fukaya, Toru Miyazaki, and Takao Kozakai, J. Mater. Sci. 26, 5420 (1991).

${ }^{13}$ K. F. Wojciechowski, Acta Metall. 6, 396 (1958); 6, 376 (1959).

${ }^{14}$ M. Schoijet and L. Muldawer, Bull. Am. Phys. Soc. 15, 360 (1970).

${ }^{15}$ S. Hosoya, J. Phys. Soc. Japan 4, 489 (1954).

${ }^{16}$ S. Matsuda, J. Phys. Soc. Japan 8, 20 (1953).

${ }^{17}$ D. C. Chrzan and L. M. Falicov, Phys. Rev. B 37, 3894 (1988). 
${ }^{18}$ R. Kikuchi, J. M. Sanchez, D. de Fontaine and H. Yamauchi. Acta Metall. 28, 651 (1990).

${ }^{19}$ C. Colinet. G. Inden and R. Kikuchi. Acta Metall. Mater. 45. 1109 (1993).

${ }^{20} \mathrm{G}$. Inden and W. Pitsh. in Phase Transformations in Materials, Fol. 5. edited by P. Haasen. (Verlag Chemie. Weinheim, 1991).

${ }^{21}$ A. J. S. Traiber, S.M. thesis, Massachusetts Institute of Technology, 1991.

${ }^{22}$ C. Wolverton and D. de Fontaine, Phys. Rev. B 49, 12351 (1994).

${ }^{23}$ C. Wolverton and D. de Fontaine, Phys. Rev. B 49, $\$ 627$ (1994).

${ }^{24} \mathrm{P}$. Turchi, Thèse de Doctorat d'Etat, Université Paris VI, 1984.

${ }^{25} \mathrm{P}$. E. A. Turchi, in Intermetallic Compounds: Principles and Practice, edited by J. H. Westbrook and R. L. Fleischer, (John Wiley \& Sons, Ltd., New York, 1995), vol. 1, Chap. 2, pp. $21-54$.

${ }^{26}$ M. Sluiter, P. Turchi, Fu Zezhong, and D. de Fontaine, Phys. Rev. Lett. 60, 716 (1988).

${ }^{27}$ M. Sluiter and P. E. A. Turchi, Phys. Rev. B 43, 12251 (1991).

${ }^{28}$ C. Colinet and A. Pasturei, Physica B 192, 238 (1993).

${ }^{29}$ G. Rubin and A. Finel, J. Phy's.: Condens. Matter. 5, 9105 (1993).

${ }^{30}$ R. M. Waterstrat, Platinum Metals Rev. 37, 194 (1993).

${ }^{31}$ R. M. Waterstrat, L. A. Bendersky, and R. Kuentzler, Mat. Res. Soc. Symp. Proc. 246, 115 (1992).

32 J. C. Slater and G. F. Koster, Phys. Rev. 94, 1498 (1954).

${ }^{33}$ H. Jone and N.F. Mott, Proc. Roy. Soc. A 162, 49 (1937).

${ }^{34}$ G. C. Fletcher, Proc. Phys. Soc. (London) A 65, 192 (1952).

${ }^{35}$ D. A. Papaconstantopoulos, Handbook of the Band Structure of Elemental Solids (Plenum, 
New York, 1986).

${ }^{30} \mathrm{~V}$. Rosato, M. Guillope, and B. Legrand, Phil. Mag. A 59. 321 (1989).

${ }^{37}$ Fabrizio Cleri and Vittorio Rosato. Phys. Rev. B 48. 22 (1993).

${ }^{3 s}$ L. Goodwin, A. J. Skinner, and P. G. Pettifor, Europhys. Lett. 9, i01 (1989).

${ }^{39}$ James L. Mercer, Jr. and M. Y. Chou, Phys. Rev. B 47, 9366 (1993).

${ }^{40}$ O. K. Andersen, O. Jepsen, and D. Glotzel, in Highlights of Condensed Matter Theory, edited by F. Bassani, F. Fermi and M. P. Tosi (North-Holland, Amsterdam, 1985), p. 59.

${ }^{41}$ Marcel H. F. Sluiter and Prabhakar P. Singh, Phys. Rev. B 49, 10918 (1994).

${ }^{42}$ Hiroyuki Shiba, Prog. Theor. Phys. 46, 77 (1971).

${ }^{43}$ Roger Haydock, in Solid State Physics V. 35, edited by H. Ehrenreich, F. Seitz, D. Turnbull, (Academic Press, New York, 19S0), p. 215.

${ }^{44}$ The Recursion Meihod and Its Applications, series in Solid State Sciences, edited by D. G. Pettifor and D. L. Weair, (Springer, Berlin, 1985).

${ }^{45}$ Paul Soven, Phys. Rev. 156, S09 (1967); B. Velicky, S. Kirkpatrick, and H. Ehrenreich, ibid 175,747 (1968).

${ }^{46}$ D. J. Chadi and M. L. Cohen, Phys Rev. B 8, 574i (19i3).

${ }^{47}$ A. Gonis, P. E. A. Turchi, X.-G. Zhang, G. M. Stocks, D. M. Nickolson, W. H. Butler, in Atomistic Simulation of Materials, edited by V. Vitek and D. J. Srolovitz, (Plenum, New York, 1989), p. 15.

${ }^{48}$ A. Gonis, P. P. Singh, P. E. A. Turchi, and X.-G. Zhang, Phys. Rev. B 51, 2122 (1995).

${ }^{49}$ F. Ducastelle, in Alloy Phase Stability, edited by: G.M. Stocks and A. Gonis, (K'luwer, Boston, 1989), p. 293. 
${ }^{50}$ A. Gonis. Green's Functions for Ordered and Disordered Systems (North-Holland, Amsterdam, 1992). Chap. 9.

51 William H. Butler, Phỹs. Rev. B 8. 4499 (1973).

${ }^{52} \mathrm{~J}$. Friedel, in Physics of Metals I: Electrons, edited by J. M. Ziman, (Cambridge University Press, Cambridge, 1969), p. 340.

${ }^{53}$ Jian-hua Xu and A. J. Freeman, Phys. Rev. B 40, 11927 (1989).

${ }^{54}$ S. M. Allen and J. IV. Cahn. Acta Metall. 20, 423 (1972).

${ }^{55}$ Ariel J. S. Traiber and S. M. Allen, Acta Metall. Mater. 40, 1403 (1992).

${ }^{56}$ F. Ducastelle, in Order and Phase Stability in Alloys, Cohesion and Structure Series V3, edited by F. R. de Boer and D. G. Pettifor, (North-Holland, Amsterdam, 1991).

${ }^{57}$ R. Kuentzler and Y. Dossmann, J. de Phys. IV, vol. 1 (no. C4), 54 (1991).

${ }^{58}$ R. M. Waterstrat, Leonid A. Bendersky, and Raymond Kuentzler, Proceedings of the International Conference on Martensitic Transformations, edited by C. M. Wayman and J. Perkins (Monterey Institute of Advanced Studies, Carmel CA, 1993), p. 545.

${ }^{59}$ C. Kittel, Introduction to Solid State Physic, 5th edition, (Wiley, New York, 1976), p. 85. 


\section{FIGURES}

FIG. 1. Hopping integrals (in $R y$ ) for $\mathrm{Zr}$ as functions of the interatomic distance as computed with the exponential fit (dashed line) to the LMTO-ASA results (square dots).

FIG. 2. On-site energies (in $\mathrm{Ry}$ ) for $\mathrm{Zr}$ as a function of $\mathrm{v}^{1 / 3}$ (where $\mathrm{v}$ is the atomic volume) in a.u. The symbols represent the LMTO results, and the broken lines are the polynomial fit.

FIG. 3. Band structure for the B2 RuZr compound along special directions of the irreducible wedge of the primitive cubic Brillouin zone. (a) from LMTO calculation; (b) using the SK parameters extracted from TB-LMTO calculations performed for the pure elements, and with the definition of "average" SK parameters for the alloy case, as defined in the text.

FIG. 4. Total DOS (in Ry/atom) of the B2 RuZr compound as a function of energy (the Fermi energy is taken as zero of energy') computed with the recursion method: (a) LMTO with no off-diagonal disorder, and (b) same approximation as explained in the caption of Fig. 3 (b).

FIG. 5. Total DOS (in $\mathrm{Ry} / \mathrm{atom}$ ) for a bcc $\mathrm{Zr}_{0.5} \mathrm{Ru}_{0.25} \mathrm{Pd}_{0.25}$ pseudo-binary alloy vs, energy (in Ry) as computed with the CCPA (solid line) and the PCPA (dashed line).

FIG. 6. On the left: real part of the self energy $\sigma^{t_{2 g}}$ corresponding to the random sublattice for the $\mathrm{Zr}_{0.5}(\mathrm{RuPd})_{0.5}$ pseudo-binary alloy computed with the CCPA. On the right: on-site energy $\tilde{\epsilon}^{t_{2} g}$ associated with the fully ordered sublattice occupied by $\mathrm{Zr}$ for the same alloy (see text). Notice the different scaling applied to the vertical axis.

FIG. 7. Total DOS (in states/Ry.atom) vs. energy (in Ry) for the binary alloys (a) RuZr, (b) PdZr. and (c) RuPd, as computed with the CPA. The Fermi energy is taken as zero of energy.

FIG. 8. Energy of mixing (in mRy/atom) vs. concentration for a.) Ru-Pd, and b) Ru-Zr (solid line) and Pd-Zr (dashed line) alloys, as computed with the CPA.

FIG. 9. First (a) and second (b) nearest neighbor EPI (in $\mathrm{mRy} / \mathrm{atom}$ ) vs. concentration for bcc-based $\mathrm{Zr}_{0.5}\left(\mathrm{Ru}_{1-\varepsilon} \mathrm{Pd}_{c}\right)_{0.5}$. Solid line: $\mathrm{Ru}-\mathrm{Zr}$; dotted line: $\mathrm{Pd}-\mathrm{Zr}$; dashed line: $\mathrm{Ru}-\mathrm{Pd}$. 
FIG. 10. Second nearest neighbor EPI (in $m R y / a t o m$ ), $V_{2}$. between $R u$ and $P d$ in the $\mathrm{Zr}_{0.5} \mathrm{Ru}_{0.25} \mathrm{Pd}_{0.25}$ alloy rs. band-filling, as computed with the GPMI applied to the fully random CPA medium. The vertical line shows the band filling that corresponds to the actual alloy.

FIG. 11. Energy of the disordered alloy $\mathrm{Zr}_{0.5}\left(\mathrm{Ru}_{1-c} \mathrm{Pd}_{c}\right)_{0.5}$, in $\mathrm{mRy} / \mathrm{atom}$, vs, concentration. Dashed line: as obtained with the PCPA; solid line: with the full CPA.

FIG. 12. Phase diagram for the pseudo-binary system $\mathrm{Zr}_{0.5}\left(\mathrm{Ru}_{1-c} \mathrm{Pd}_{c}\right)_{0.5}$ computed with the CVM in the simple cube approximation. The dashed line correspond to the spinodal line of decomposition.

FIG. 13. Electronic specific heat coefficient, in $\mathrm{mJ}(\mathrm{g} \cdot \mathrm{at})^{-1} \cdot \mathrm{K}^{-2}$, as a function of Pd-concentration for the pseudo-binary $\mathrm{Zr}_{0.5}\left(\mathrm{Ru}_{1-c} \mathrm{Pd}_{c}\right)_{0.5}$ alloy (from Ref. $5 \bar{i}$ ).

FIG. 14. DOS's (in states/Ry.atom), at the Fermi energy, as a function of Pd-concentration, for a) $(\mathrm{ZrRu})_{1-c}^{B 2}(\mathrm{ZrPd})_{c}^{B 2}$ (see text), and b) $\mathrm{Zr}_{0.5}\left(\mathrm{Ru}_{1-c} \mathrm{Pd}_{c}\right)_{0.5}$ from PCPA. 


\section{TABLES}

TABLE I. Tight-binding fitting parameters $\mathcal{A}_{i}^{h}$ and $\mathcal{P}_{i}^{h}$ for Zr. Ru and Pd. see Eq. 2.2. $\mathcal{A}^{h}$ and $\mathcal{P}^{h}$ are expressed in $R y$ and $(\text { a.u. })^{-1}$, respectively.

\begin{tabular}{ccccccc}
\hline & \multicolumn{2}{c}{$\mathrm{Zr}$} & \multicolumn{2}{c}{$\mathrm{Ru}$} & \multicolumn{2}{c}{$\mathrm{Pd}$} \\
SK Parameter $(h)$ & $\mathcal{A}^{h}$ & $\mathcal{P}^{h}$ & $\mathcal{A}^{h}$ & $\mathcal{P}^{h}$ & $\mathcal{A}^{h}$ & $\mathcal{P}^{h}$ \\
\hline$s s \sigma$ & -74.8759 & 1.2668 & -113.1613 & 1.3912 & -112.0270 & 1.3823 \\
$p p \sigma$ & 57.7912 & 1.1981 & 137.4739 & 1.3378 & 137.7712 & 1.3240 \\
$p p \pi$ & -54.1653 & 1.5654 & -289.4552 & 1.8810 & -188.6586 & 1.7817 \\
$d d \sigma$ & -40.4902 & 1.0714 & -39.9683 & $1.17 i 4$ & -28.3505 & 1.1708 \\
$d d \pi$ & 208.5139 & 1.5235 & 293.2882 & 1.7263 & 204.3557 & 1.7090 \\
$d d \delta$ & -373.1065 & 2.0514 & -619.6101 & 2.3208 & -421.0329 & 2.2903 \\
$s p \sigma$ & 74.5814 & 1.2541 & 142.0822 & 1.3885 & 141.3045 & 1.3765 \\
$s d \sigma$ & -62.4407 & 1.1972 & -76.4761 & 1.3148 & -64.1410 & 1.3068 \\
$p d \sigma$ & -47.8802 & 1.1358 & -73.5461 & 1.2592 & -61.8225 & 1.2484 \\
$p d \pi$ & 129.0867 & 1.5787 & 358.0203 & 1.8423 & 241.0660 & 1.7833 \\
\hline \hline
\end{tabular}


TABLE II. Polynomial coefficients for the on-site energies, see Eq. 2.6.

\begin{tabular}{|c|c|c|c|}
\hline \multicolumn{4}{|c|}{$\mathrm{Zr}$} \\
\hline$\lambda$ & $A[R y]$ & $\left.B[R y \text { (a.u. })^{-1}\right]$ & $\left.C \cdot[R] ;(\text { a.u. })^{-2}\right]$ \\
\hline $\mathcal{s}$ & 12.264350 & -3.181079 & 0.206417 \\
\hline$p$ & 1.413209 & 0.073624 & -0.033483 \\
\hline$t_{2 g}$ & 7.620164 & -1.913354 & 0.119266 \\
\hline$e_{g}$ & 7.078303 & -1.781268 & 0.110827 \\
\hline
\end{tabular}

$\mathrm{Ru}$

\begin{tabular}{llrc}
$\lambda$ & $\mathrm{A}[\mathrm{Ry}]$ & $\mathrm{B}\left[\mathrm{Ry}(\text { a.u. })^{-1}\right]$ & $\mathrm{C}\left[\mathrm{Ry}(\text { a.u. })^{-2}\right]$ \\
\hline$s$ & 9.750855 & $-2 . i 37415$ & 0.188767 \\
$p$ & 9.586590 & -2.544969 & 0.169262 \\
$t_{2 g}$ & 6.973325 & -2.030726 & 0.141034 \\
$e_{g}$ & 6.492289 & -1.904228 & 0.132484 \\
\hline
\end{tabular}

$\mathrm{Pd}$

\begin{tabular}{cccc}
$\lambda$ & $\mathrm{A}[\mathrm{Ry}]$ & $\mathrm{B}\left[\mathrm{Ry}(\text { a.u. })^{-1}\right]$ & $\mathrm{C}\left[\mathrm{Ry}(\text { a.u. })^{-2}\right]$ \\
\hline$s$ & 7.608464 & -2.156563 & 0.148761 \\
$p$ & 8.526845 & -2.294863 & 0.153963 \\
$t_{2 g}$ & 5.163944 & -1.578082 & 0.111592 \\
$e_{g}$ & 4.789320 & -1.478577 & 0.104818 \\
\hline \hline
\end{tabular}

TABLE III. Effective pair interactions (in $\mathrm{mRy} / \mathrm{atom}$ ) for the bcc-based binary alloy at equiatomic composition, as computed with the CPA-GPM formalism.

\begin{tabular}{lccccc}
\hline shell & 1 & 2 & 3 & 4 & 5 \\
\hline$V^{\text {RuZr }}$ & 31.18 & 1.75 & 0.80 & 0.81 & -2.37 \\
$V^{\text {PdZr }}$ & 20.28 & 1.13 & 1.20 & 1.59 & -2.13 \\
$V^{\text {RuPd }}$ & -1.57 & -2.51 & -0.14 & -0.28 & 0.57 \\
\hline
\end{tabular}



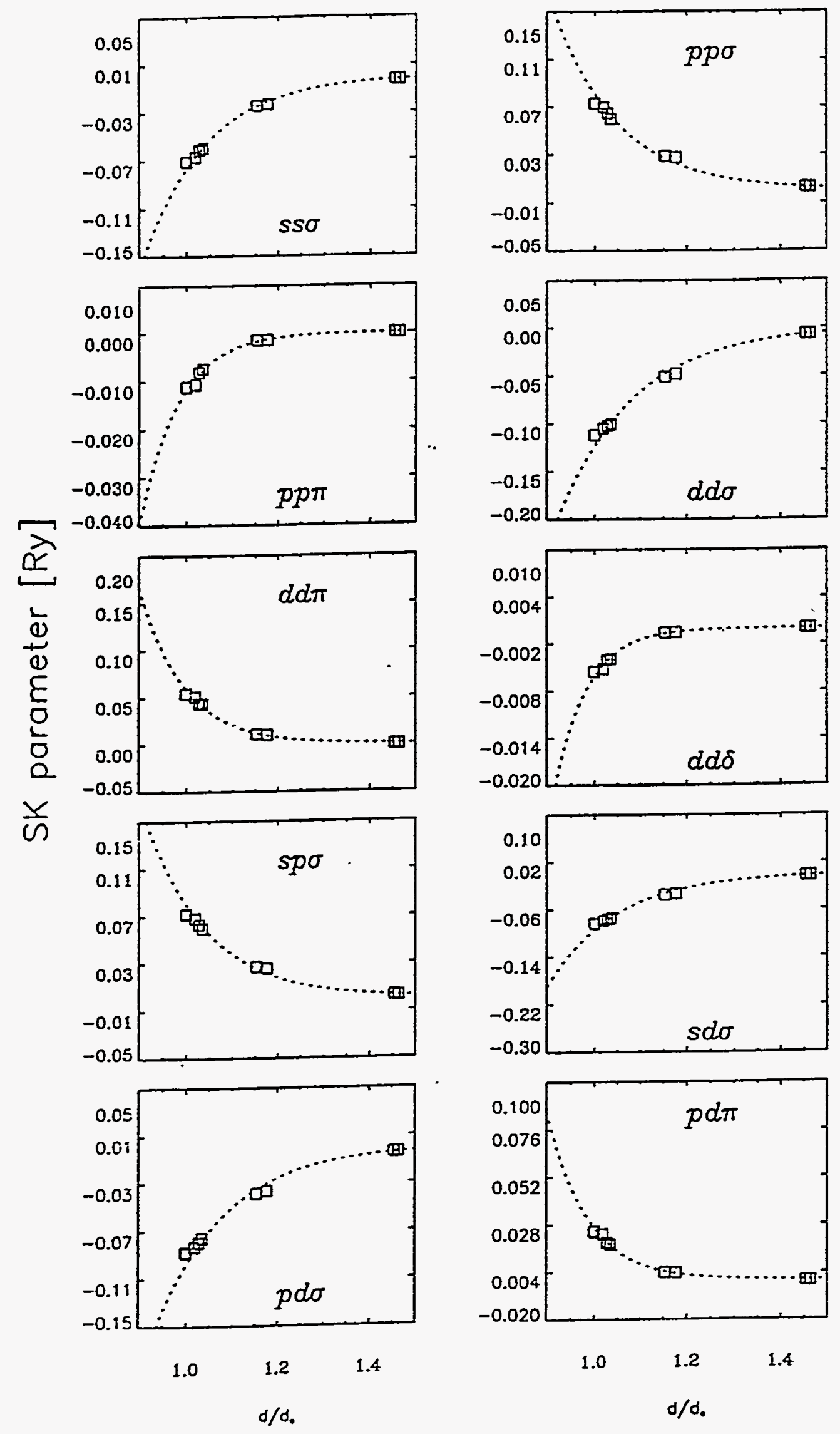

Figure 1 


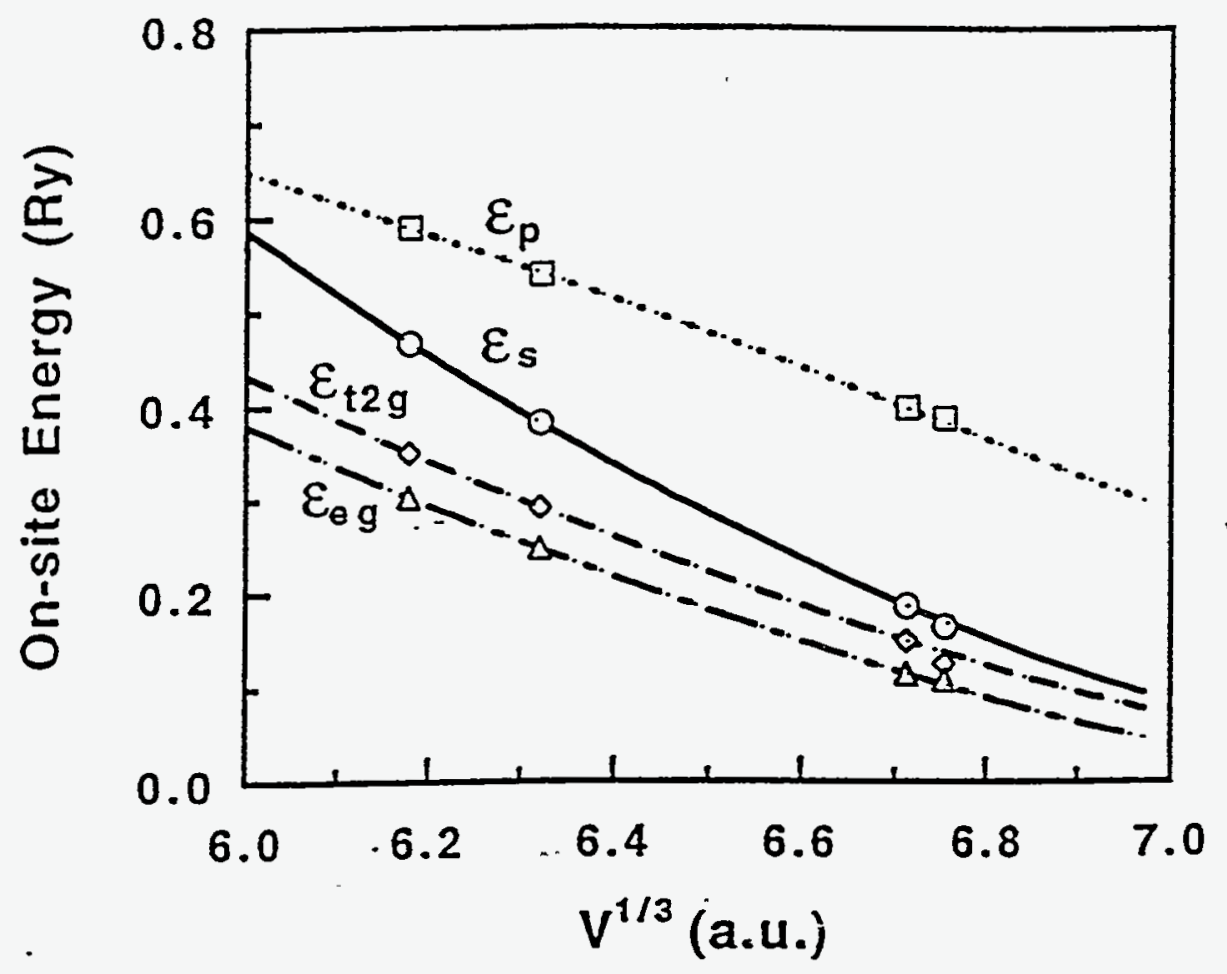

Figure 2 


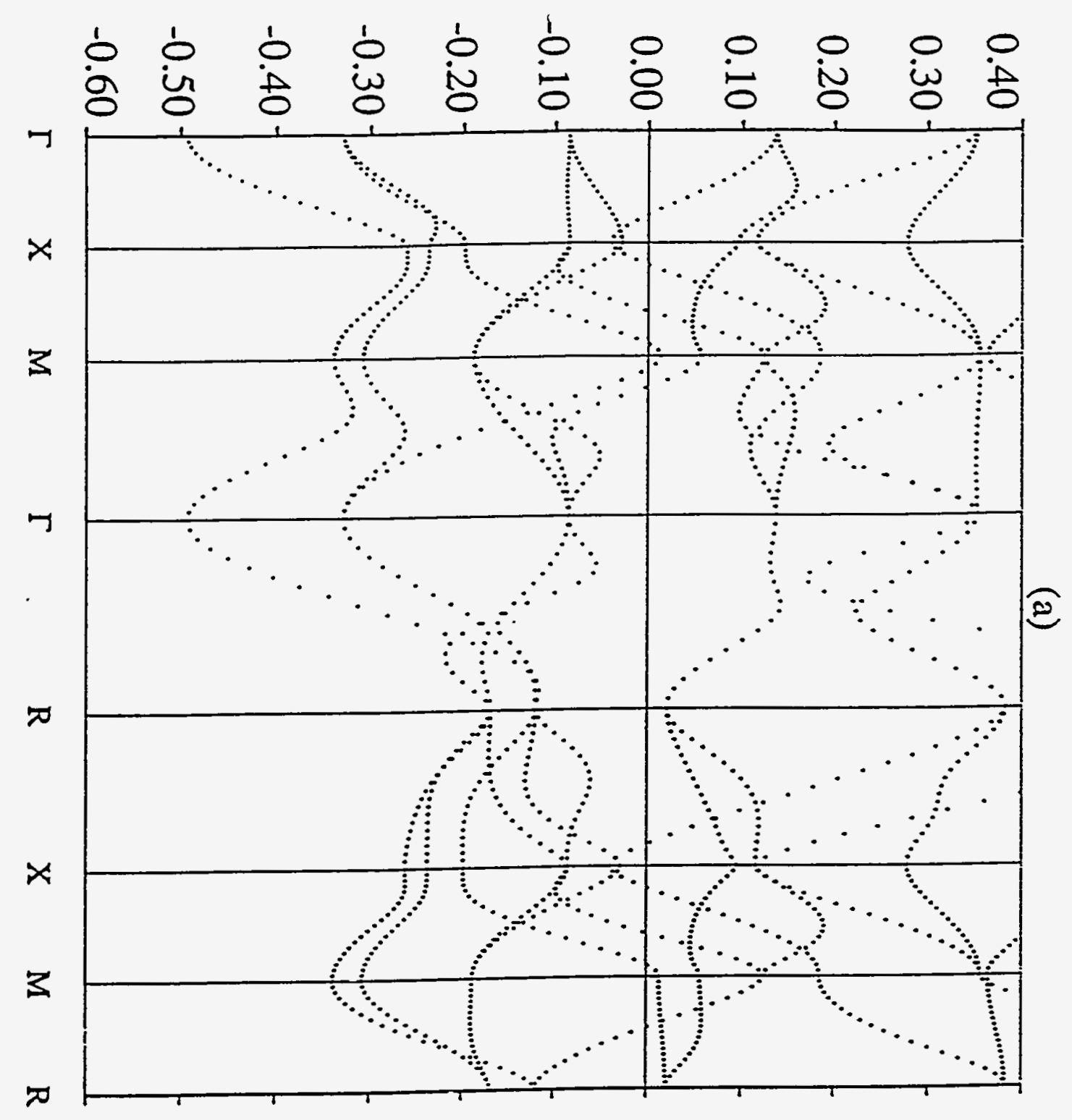


$\mathrm{E}(\mathrm{Ky})$

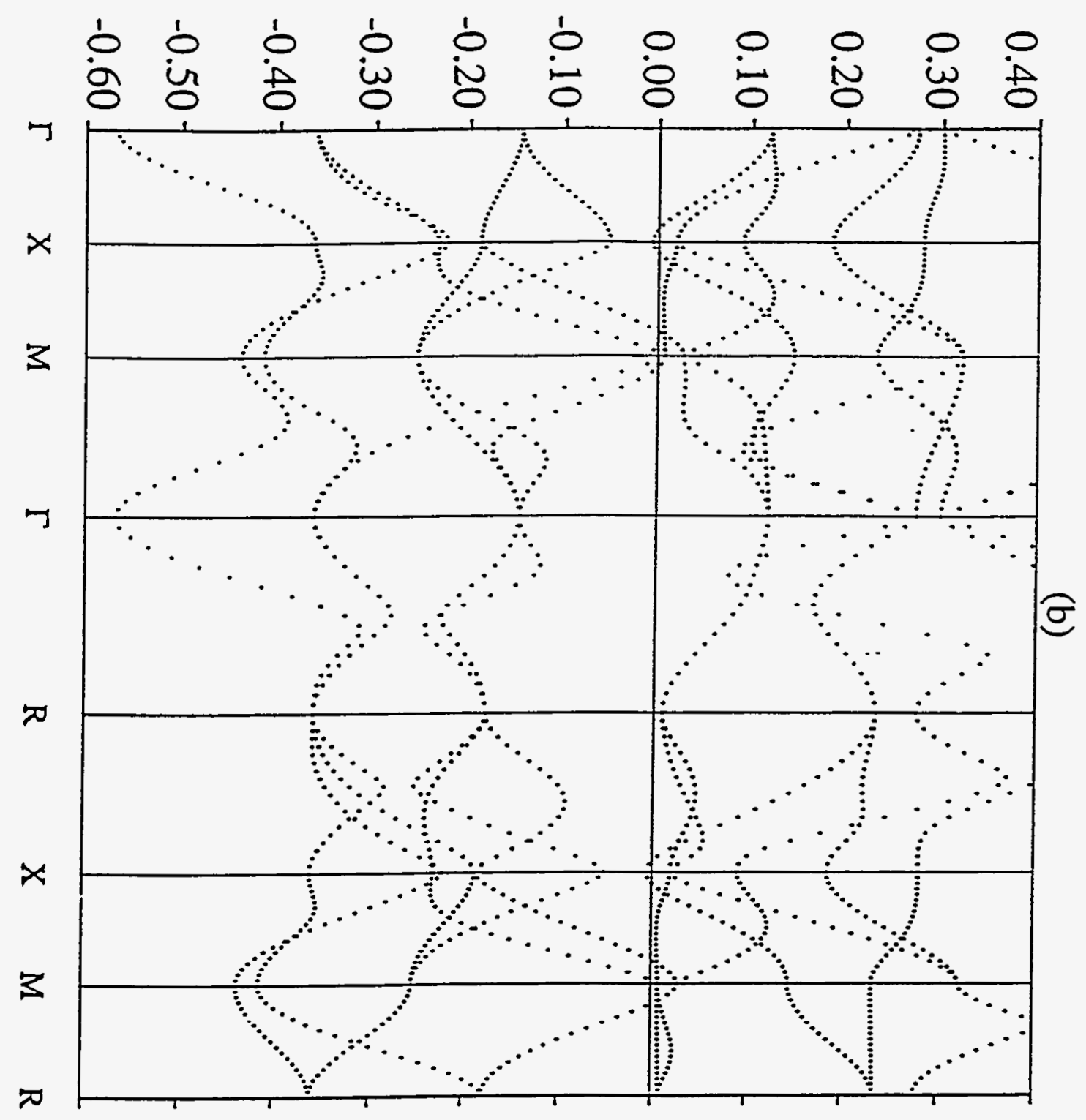

Figure $3 b$ 

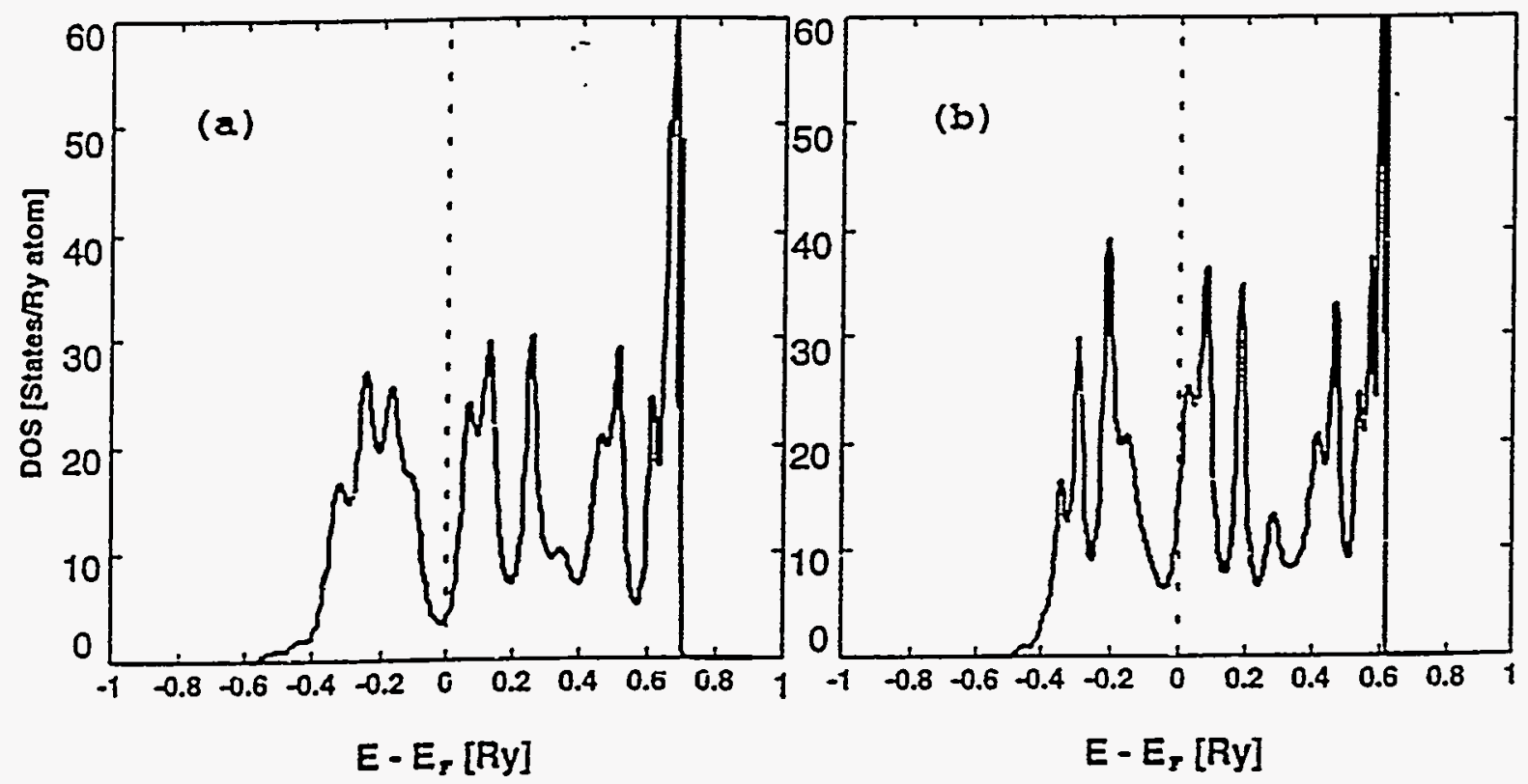

Figure 4 
DOS [States/ RY site]

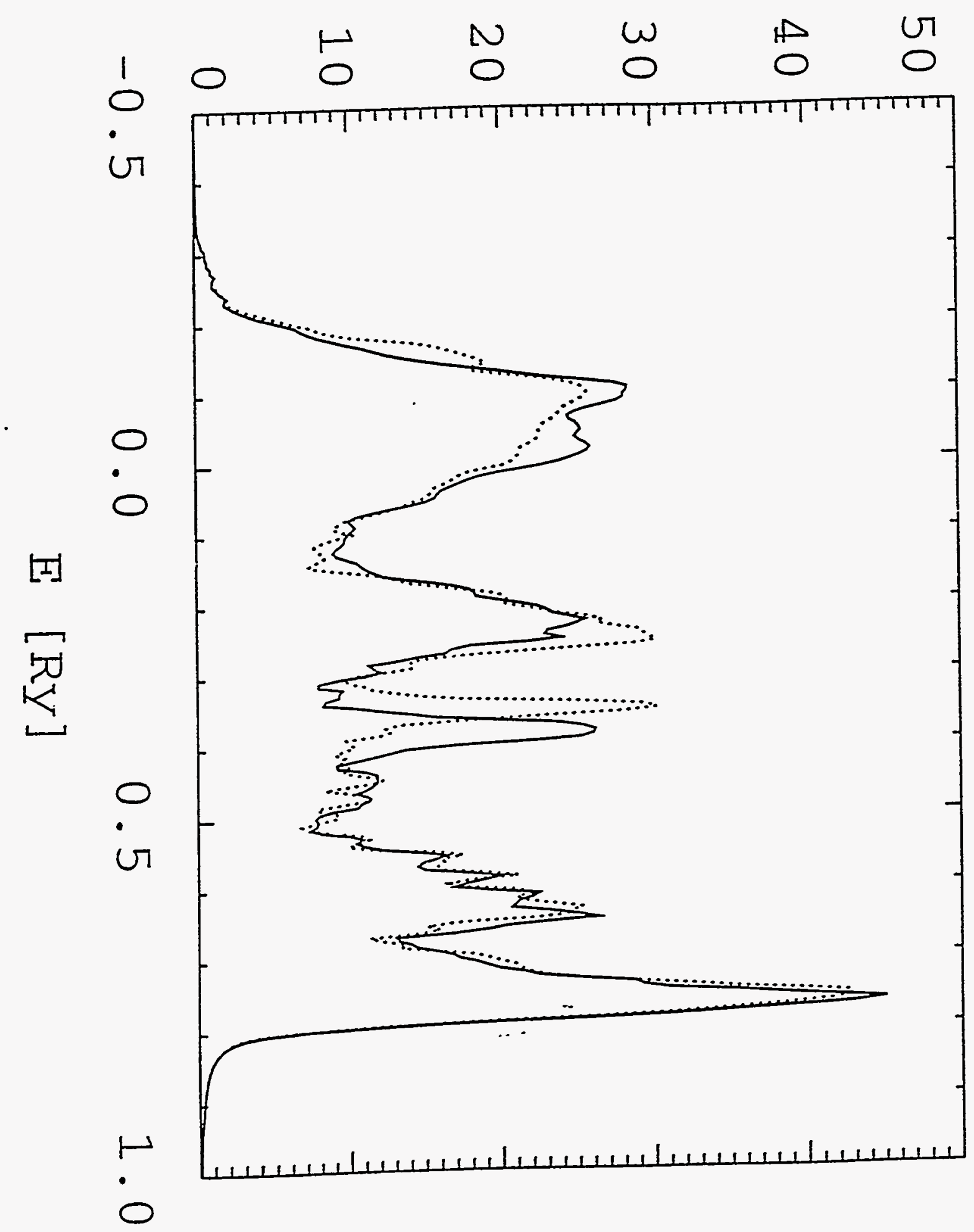



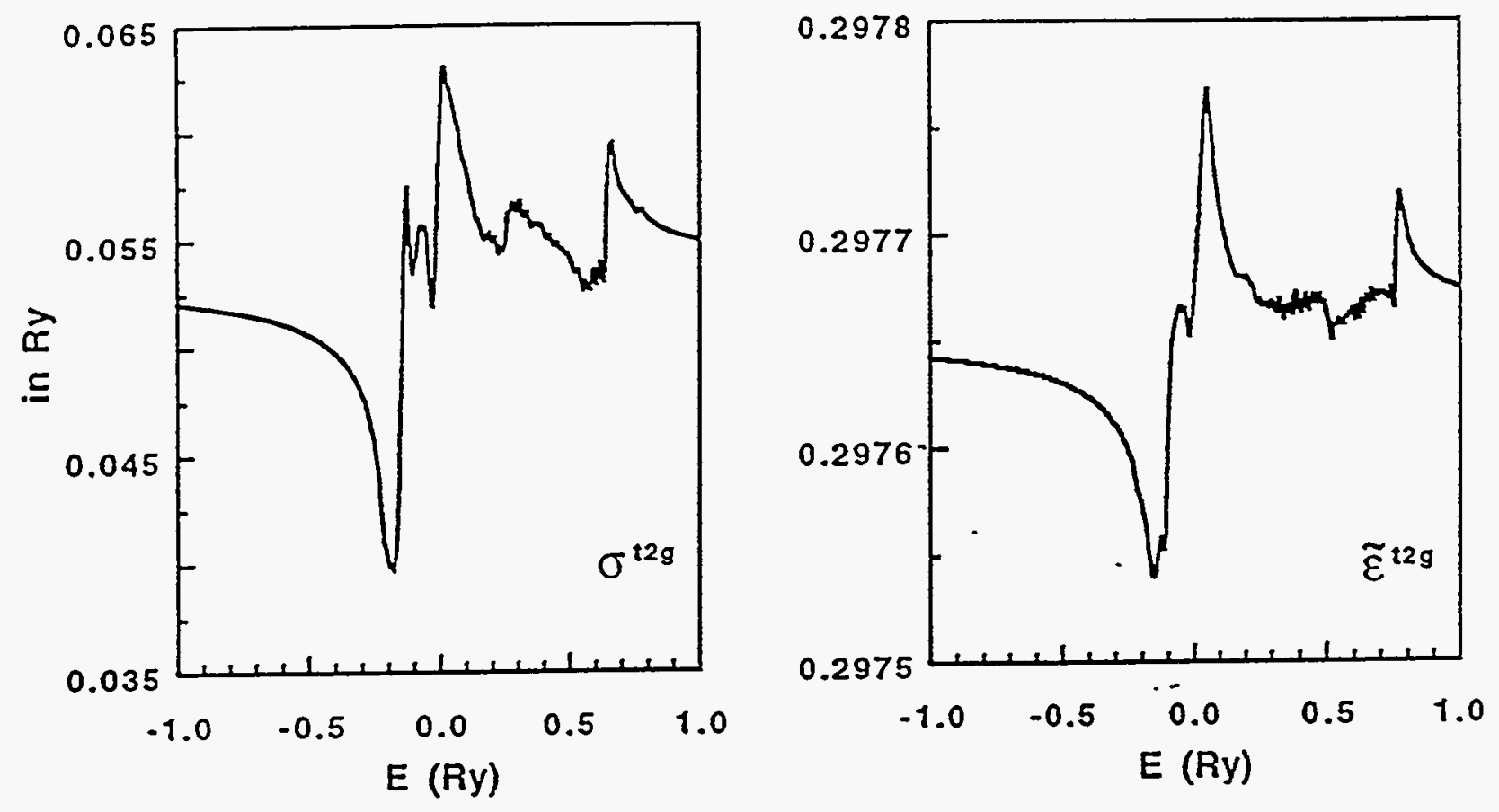

Figure 6 


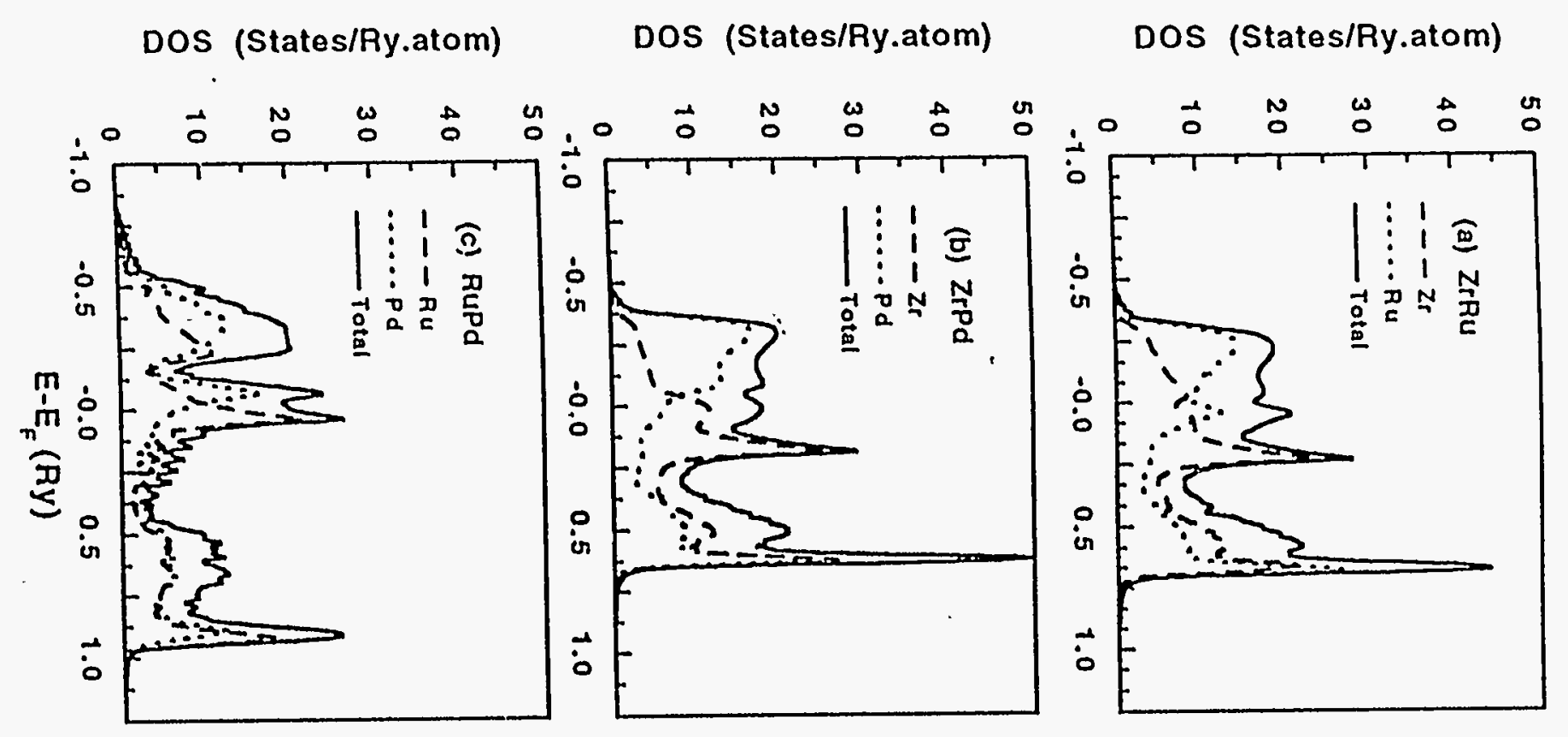



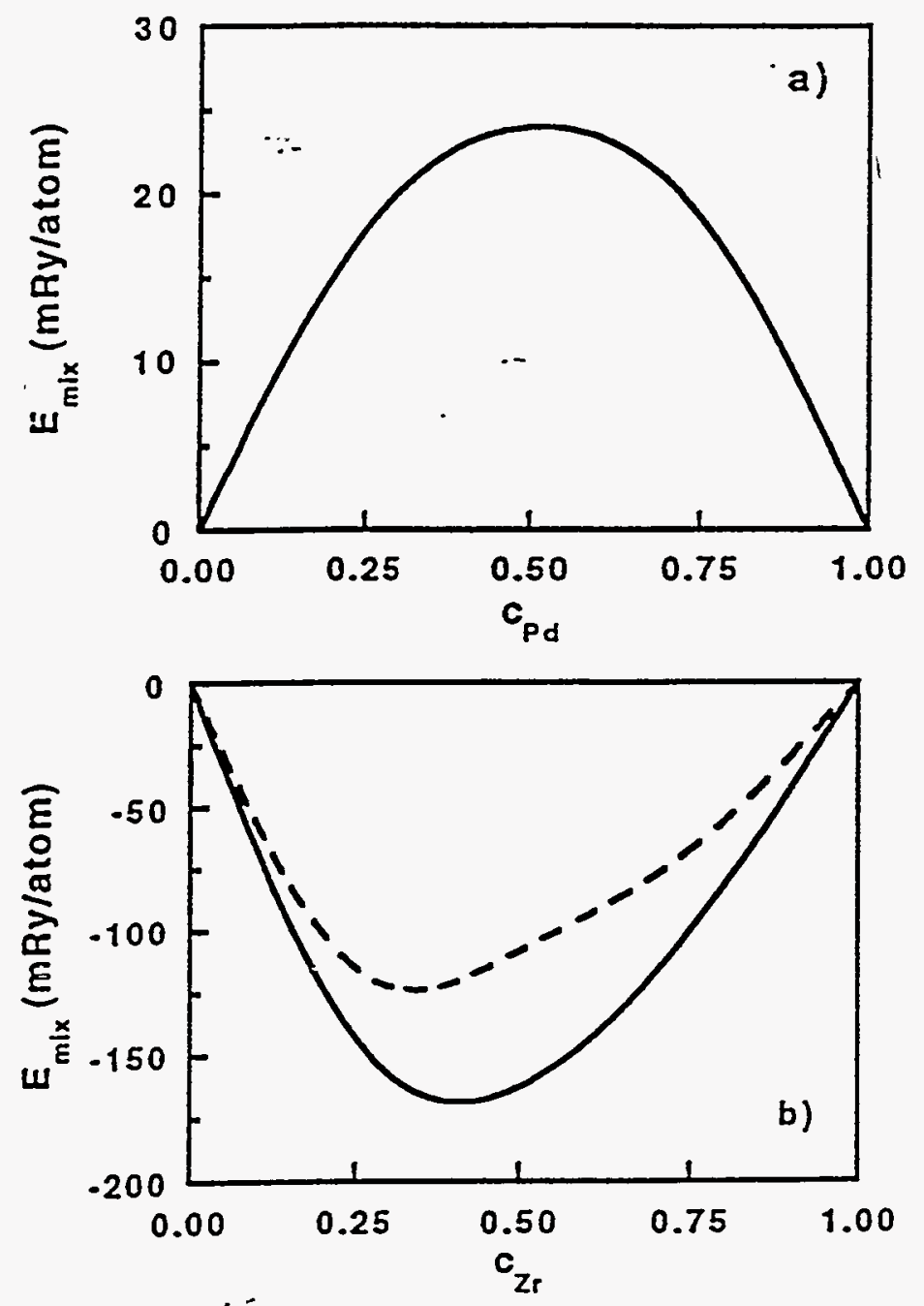

Figure 8 

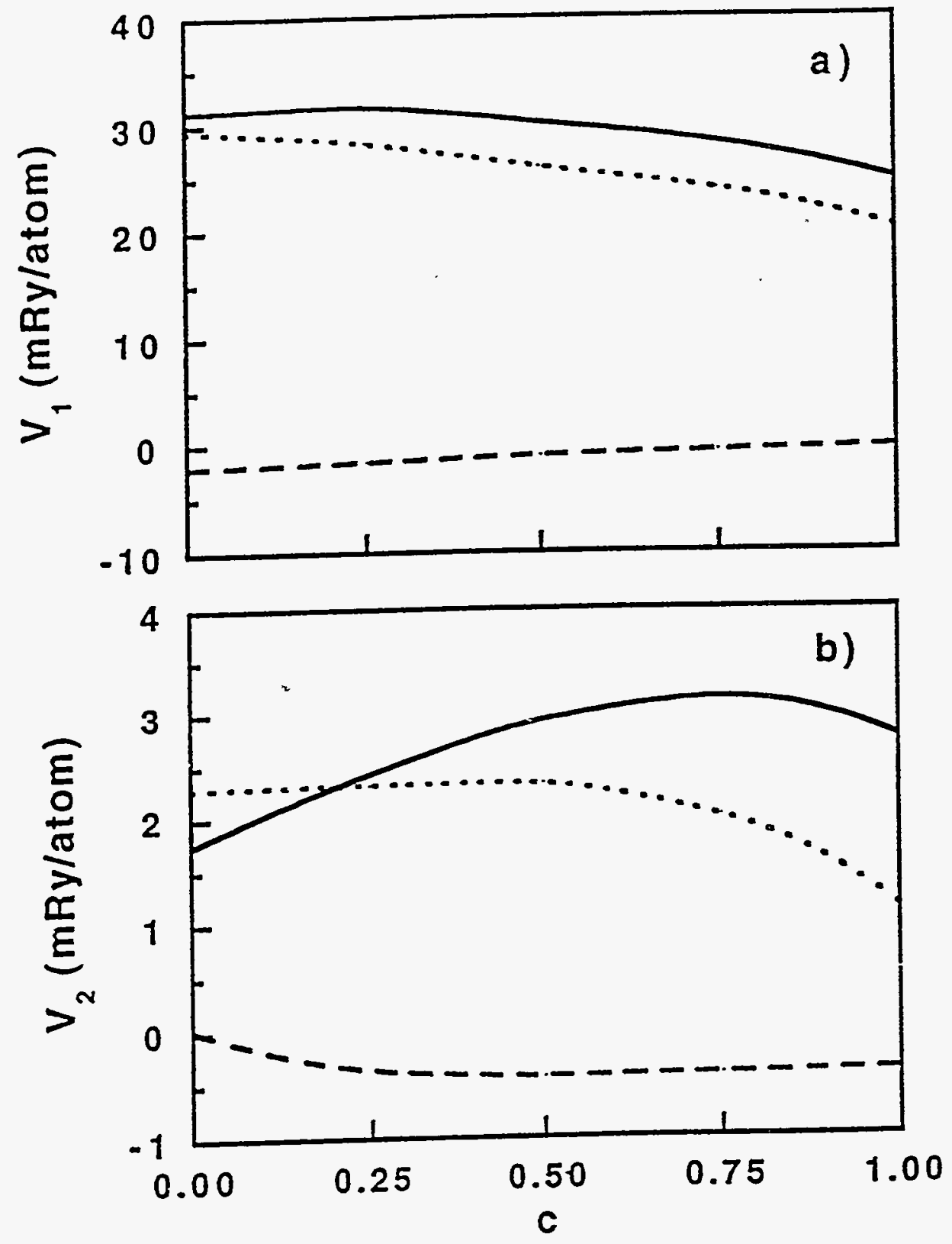

Figure 9 


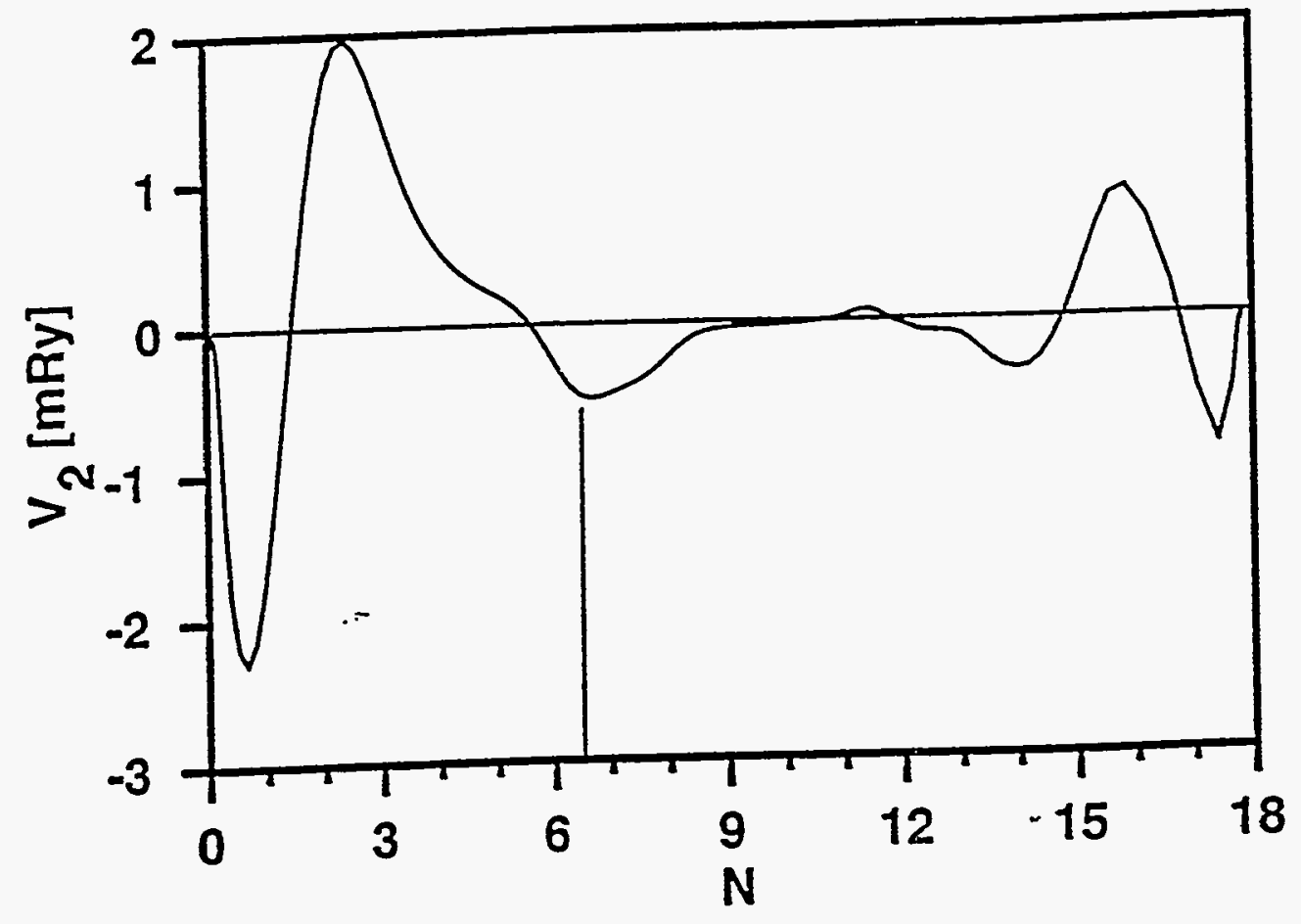

Figure 10 


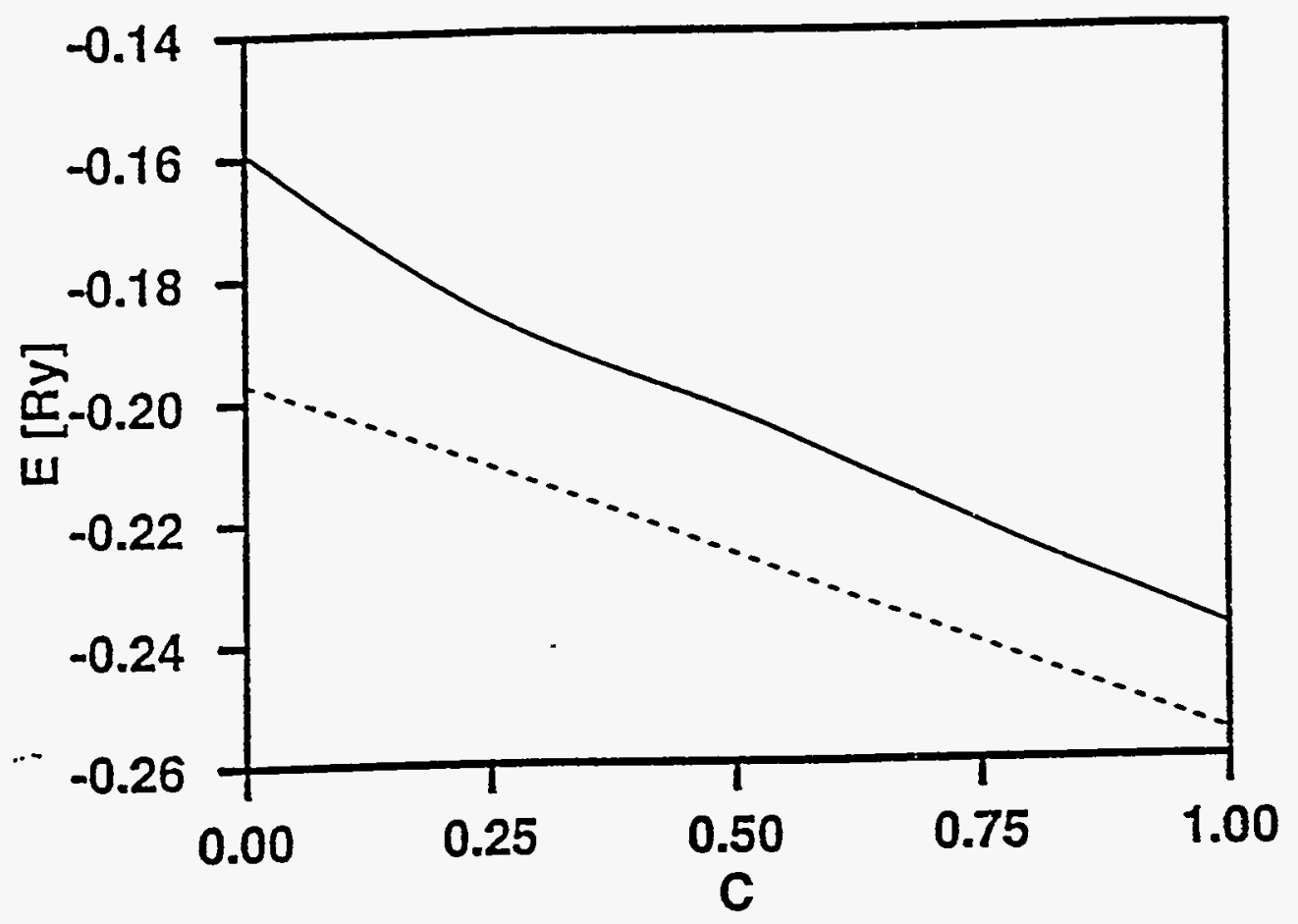

Figure 11 


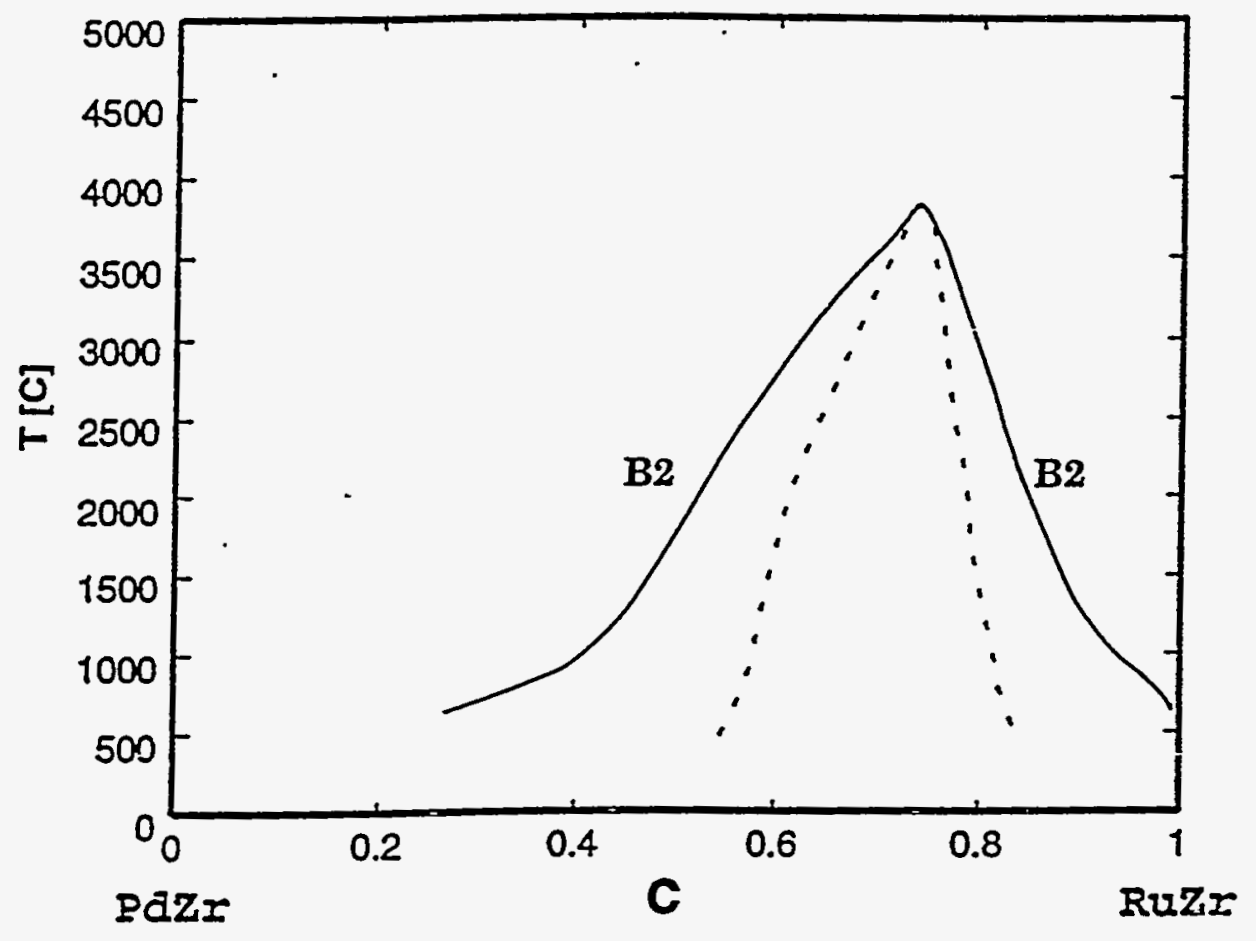

Figure 12 


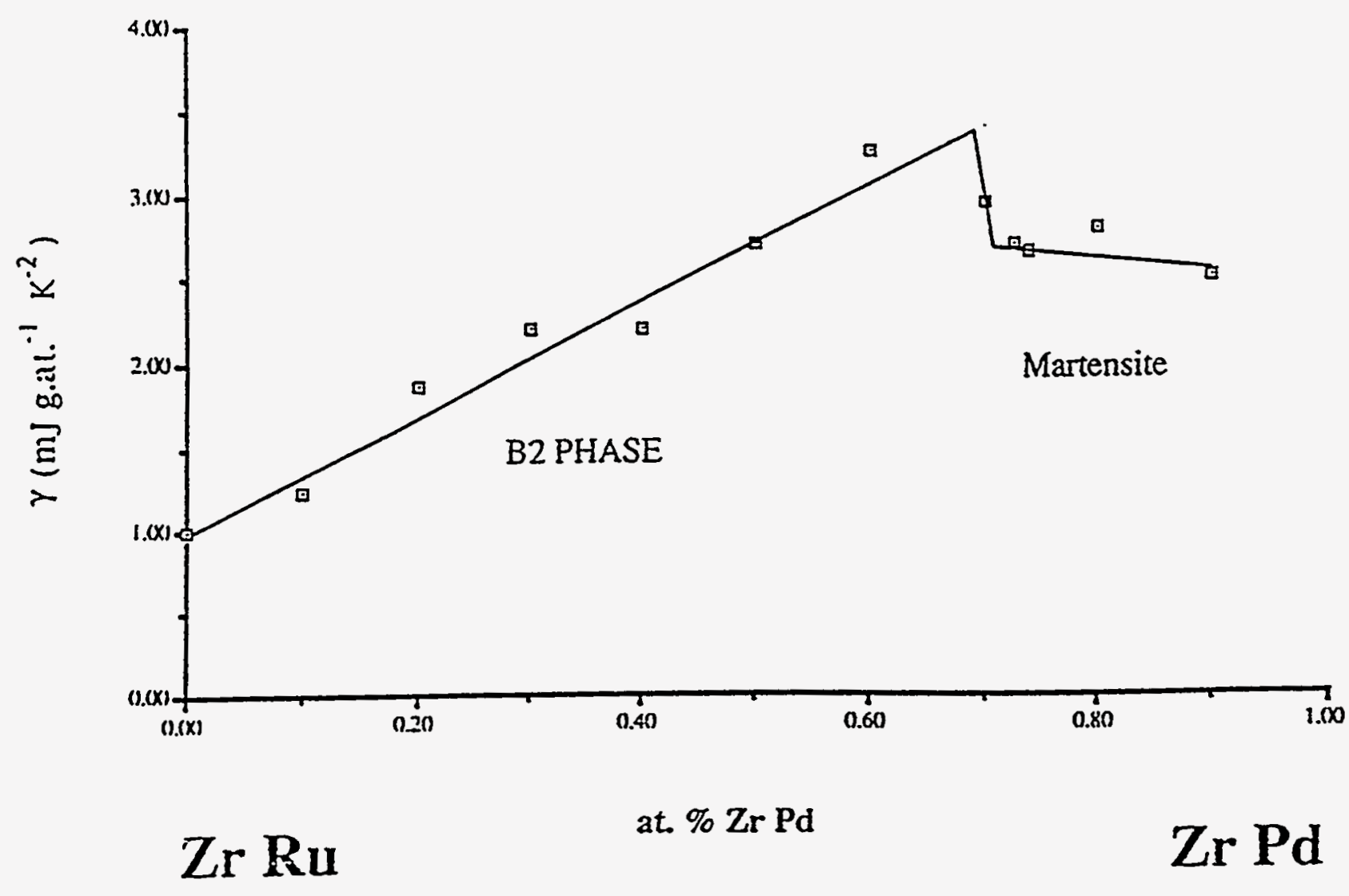

Figure 13 


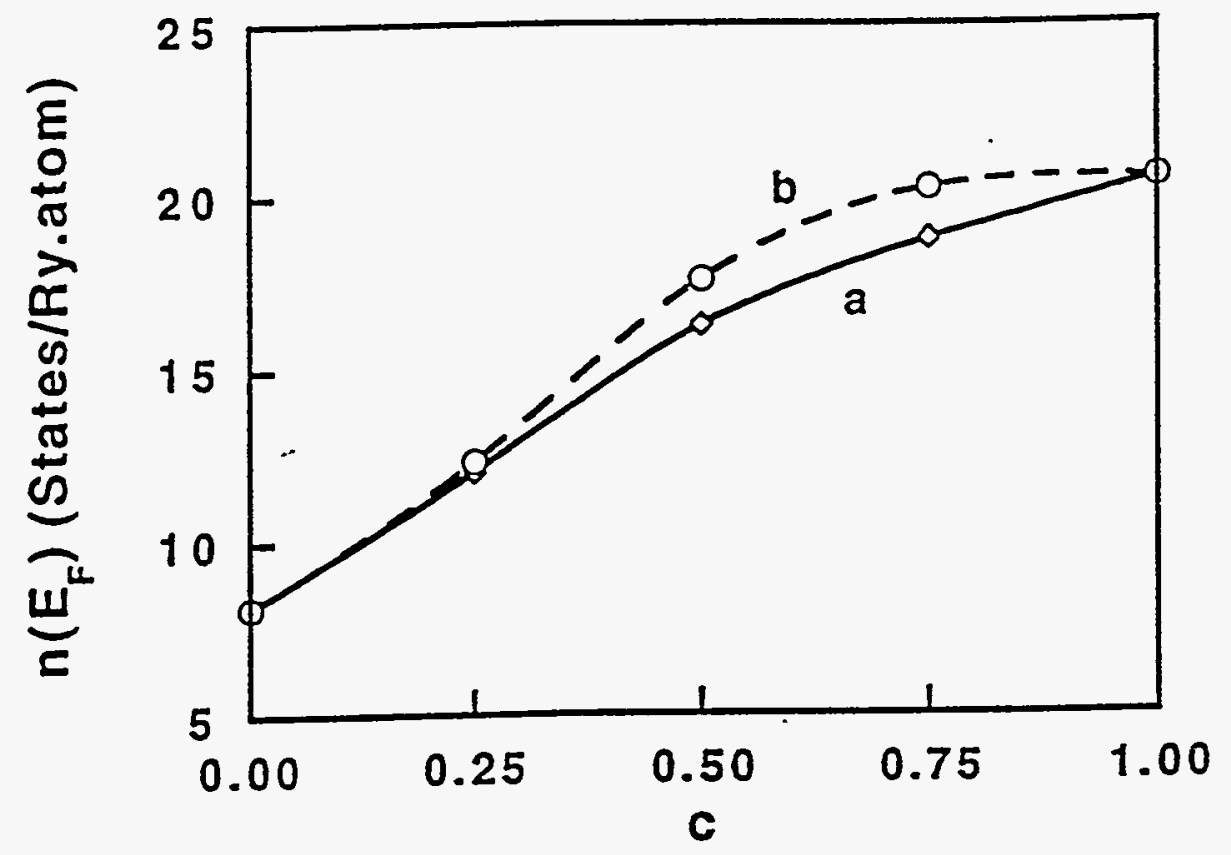

Figure 14 